\title{
REVIEW
}

\section{Marine mammal bycatch in gillnet and other entangling net fisheries, 1990 to 2011}

\author{
Randall R. Reeves ${ }^{1}$, Kate McClellan ${ }^{2,3, *}$, Timothy B. Werner ${ }^{2,4}$ \\ ${ }^{1}$ Okapi W ildlife Associates, 27 Chandler Lane, Hudson, Quebec J0P 1H0, Canada \\ ${ }^{2}$ Consortium for Wildlife Bycatch Reduction, John H. Prescott Marine Laboratory, New England Aquarium, \\ Central Wharf, Boston, Massachusetts 02118, USA \\ ${ }^{3}$ Department of Environmental Conservation, University of Massachusetts Amherst, Amherst, Massachusetts 01003, USA \\ ${ }^{4}$ Department of Biology, Boston University, 5 Cummington Mall, Boston, Massachusetts 02115, USA
}

\begin{abstract}
Since the 1970s the role of fishery bycatch as a factor reducing, or limiting the recovery of, marine mammal populations has been increasingly recognized. The proceedings of a 1990 International Whaling Commission symposium and workshop summarized fishery and bycatch data by region, fishery, and species, and estimated the significance of the 'impacts' of bycatch in passive gear on all cetacean species and subspecies or geographically defined populations. A global review of pinniped bycatch in 1991 concluded that incidental mortality in passive gear had contributed to declines of several species and populations. Here we update the information on cetacean gillnet bycatch, assess bycatch data on marine mammals other than cetaceans (i.e. pinnipeds, sirenians, and 2 otter species), determine where important data gaps exist, and identify species and populations known or likely to be at high risk from bycatch in gillnets. We found that at least $75 \%$ of odontocete species, $64 \%$ of mysticetes, $66 \%$ of pinnipeds, and all sirenians and marine mustelids have been recorded as gillnet bycatch over the past 20-plus years. Cetacean bycatch information in some areas has improved, facilitating our ability to identify species and populations at high risk, although major gaps remain. Understanding of the scale of pinniped and sirenian bycatch has also improved, but this bycatch remains poorly documented, especially at the population level. This study reveals how little is known about marine mammal bycatch in gillnets in much of the world. Even as other significant threats to marine mammals have become better documented and understood, bycatch remains a critical issue demanding urgent attention if there is to be any hope of preventing further losses of marine mammal diversity and abundance, and of protecting, or restoring, ecological health.
\end{abstract}

KEY WORDS: Bycatch $\cdot$ Entanglement $\cdot$ Gillnets $\cdot$ Marine mammals

\section{INTRODUCTION}

Bycatch (incidental mortality and injury in fishing gear; see 'Materials and methods' for a more exact definition) has been increasingly recognized since the 1970 s as a factor limiting or reducing marine mammal populations (Mitchell 1975, Hofman 1995, Read 2005, 2008). A benchmark for this recognition was the 1990 Symposium and Workshop on the Mortality of Cetaceans in Passive Fishing Nets and Traps organized and convened by the Scientific Committee of the International Whaling Commission (IWC) (Perrin et al. 1994). The published proceedings of that event included a global summary of fishery and bycatch data by region, fishery, and species, as well as an experts' evaluation of the 'impacts' of bycatch on many cetacean species and geographically defined populations (IWC 1994). The workshop report contained a series of recommendations related to bycatch documentation, mitigation, and monitor- 
ing. Six species or populations were highlighted as urgently needing action to reduce unsustainable bycatch: the Yangtze River dolphin or baiji Lipotes vexillifer, the Gulf of California porpoise or vaquita Phocoena sinus, coastal populations of humpback dolphins Sousa sp. and bottlenose dolphins Tursiops sp. in KwaZulu-Natal (South Africa), striped dolphins Stenella coeruleoalba in the Mediterranean Sea, and harbor porpoises Phocoena phocoena in the western North Atlantic. Three other populations were 'of particular concern' because of large known bycatch levels thought to be unsustainable: dusky dolphins Lagenorhynchus obscurus in the eastern South Pacific (specifically Peru), northern right whale dolphins Lissodelphis borealis in the central North Pacific, and sperm whales Physeter macrocephalus in the Mediterranean Sea.

In a separate effort, Woodley \& Lavigne (1991) reviewed the literature for information on bycatch of pinnipeds and concluded that incidental mortality in passive gear had contributed to declines in populations of northern fur seals Callorhinus ursinus and harbor seals Phoca vitulina in the North Pacific and harp seals Pagophilus groenlandica in the Barents Sea. They also believed that mortality in commercial trawl fisheries was at least partly responsible for a decline in Steller sea lions Eumetopias jubatus and that bycatch had had 'detrimental impacts' on New Zealand sea lions Phocarctos hookeri, harbor seals Phoca vitulina off Newfoundland and Alaska, gray seals Halichoerus grypus in the eastern Baltic Sea, and endangered Mediterranean and Hawaiian monk seals Monachus schauinslandi and M. monachus, respectively.

Over the 20-plus years since 1990 much has changed. One of the cetacean species singled out in 1990 as being in great peril, the baiji, is now probably extinct (Turvey et al. 2007). The vaquita has continued to decline as a result of unsustainable bycatch in fishing gear (Rojas-Bracho et al. 2006, JaramilloLegorreta et al. 2007); it is now widely regarded as the world's most endangered cetacean species. Dusky dolphins have continued to be killed in Peru, and the subspecies there (Lagenorhynchus obscurus posidonia) may still be declining as a result, despite a series of legislative measures intended to reduce mortality from the deliberate targeting of cetaceans (Van Waerebeek et al. 2002, Mangel et al. 2010). Similarly, humpback dolphins and bottlenose dolphins have continued to be subjected to incidental mortality in anti-shark nets off KwaZulu-Natal (Peddemors et al. 1997, Peddemors 1998, Best 2007), with no clear assessment since the late 1980s/early 1990s of the potential population-level impacts (Ross et al. 1989, Cockcroft 1990, Cockcroft et al. 1991, 1992). Finally, sperm whales Physeter macrocephalus in the Mediterranean Sea are thought to number only in the 100 s, and they are still dying in drift nets (largely illegal since 2002 when the European Union imposed a total ban on driftnetting by member states); a major difference now is that the evidence for demographic isolation of Mediterranean sperm whales is much stronger than it was in 1990 (Notarbartolo di Sciara et al. 2006, Engelhaupt et al. 2009).

At least 2 of the cetacean populations highlighted in 1990 would probably not be ranked as being of such high concern today, at a global scale, as they were then. The United Nations ban on the use of large-scale, high-seas driftnets, which took effect at the end of 1992 (Northridge \& Hofman 1999), greatly reduced the driftnet mortality of northern right whale dolphins. Although gillnetting of billfish, sharks, squid, and tuna inside the exclusive economic zones (EEZs) of some North Pacific countries probably continue to kill 100s of these dolphins each year, the total number of living right whale dolphins remains fairly high: there were estimated to be 10000 s to 100000 s in the central North Pacific in the early 1990s (Buckland et al. 1993) and about 8000 in the United States EEZ in 2005 to 2008 (Carretta et al. 2011). Given the ongoing driftnet ban on the high seas and these relatively high estimates of abundance, the need for conservation measures directed at northern right whale dolphins seems less urgent now than it did 2 decades ago.

Further, harbor porpoises have been found to be much more abundant in the western North Atlantic than was assumed in 1990, when the 'best available estimates' ranged between 8000 and 15300 (northeastern USA, Bay of Fundy, and southwestern Nova Scotia region; IWC 1994, p. 31) compared with a 2006 estimate of 89054 (coefficient of variation, $\mathrm{CV}=0.47$ ) (Gulf of Maine/Bay of Fundy stock) (Waring et al. 2011). Annual porpoise bycatch in gillnets and other passive gear in this region were estimated at 300 to 800 in 1990 (IWC 1994, p. 25) compared with an estimate of total annual human-caused mortality in 2004 to 2008 of $928+(\mathrm{CV}=0.16)$ in all United States and Canadian fisheries in the Gulf of Maine and Bay of Fundy (Waring et al. 2011). Although this high number of annual porpoise deaths is cause for ongoing concern, the situation appears less grave than it did in 1990 in terms of sustainability (Orphanides \& Palka 2013, this Theme Section, Read 2013, this Theme Section). The situation for striped dolphins in the Mediterranean Sea is broadly similar 
to that of harbor porpoises in the western North Atlantic, with the caveat that besides continuing to sustain considerable bycatch, they have been strongly affected since 1990 by a series of die-offs from disease (Aguilar \& Gaspari 2012). Although 1000s of striped dolphins are still killed in the Mediterranean each year in drift nets (e.g. Tudela et al. 2005), they remain the most abundant cetaceans in the region, numbering at least 10000 (Aguilar \& Gaspari 2012).

In a recent authoritative global review of pinniped conservation problems (Kovacs et al. 2012), bycatch was identified as a primary threat to the Critically Endangered Saimaa ringed seal Pusa hispida saimensis and as an 'acute threat' to 3 Endangered taxa - the Australian sea lion Neophoca cinerea, the Caspian seal Phoca caspica, and the Ladoga ringed seal Pusa hispida ladogensis. Although the gears involved were not specified in all cases, we know that the threat to these 4 taxa comes principally from gillnets. Kovacs et al. (2012) also acknowledged that even though the absolute level of gillnet bycatch may be relatively low in the case of the 2 Critically Endangered monk seals, 'any human-caused mortality (of those 2 species) is a concern.'

In general, it is clear that despite the actions taken since 1990 by international, regional, and national regulatory bodies to limit and reduce bycatch, it is still a potent global threat to marine mammals. As summarized above, the problems recognized in 1990 continue to fester. Now though, as a result of greatly expanded research and monitoring, new problem species, populations, fisheries, and regions are recognized. Even as other significant threats to marine mammal populations have become better documented and understood over the past 2 decades underwater noise, ship strikes, reductions in prey populations, toxic algal blooms, epizootic disease, and various environmental changes related to global climate change-bycatch has retained its prominence as a critical issue demanding urgent attention if there is to be any hope of preventing further losses of marine mammal diversity and abundance and protecting (or restoring) ecological health.

Three particular aspects of the bycatch problem that were known to exist in 1990 have become much better understood and are now more widely acknowledged. These are: (1) the large-scale mortality of marine mammals in other types of fishing gear besides gillnets (e.g. trawls, purse seines, fish traps, longlines) (Read et al. 2006); (2) the large-scale but poorly documented mortality of marine mammals in non-industrial fisheries, i.e. in what are usually referred to as small-scale artisanal fisheries, espe- cially in developing countries (Moore et al. 2010); and (3) what Read (2008) described as a 'transition from bycatch to market value,' that is, animals that were formerly caught only incidentally and were discarded now have market (or household) value and thus have become part of the targeted catch.

This present paper is limited to 4 main objectives, as follows: (1) to update the information summarized in the 1990 workshop report on cetacean bycatch (IWC 1994); (2) to update bycatch data on marine mammals other than cetaceans (i.e. pinnipeds, sirenians, and 2 otter species); (3) to determine where important temporal, spatial, or taxonomic data gaps exist; and (4) to identify species and populations known or likely to be at greatest risk from bycatch in gillnets.

\section{MATERIALS AND METHODS}

\section{Study area}

The project's geographical scope encompassed the global distribution of marine mammals, customarily defined to include cetaceans, pinnipeds, and sirenians living partly or entirely in freshwater systems (e.g. Asian and South American rivers, Lake Baikal in Russia) and the 2 otter species that live exclusively in marine environments. All species and areas were of equal interest, although, as will be evident, our data and results were strongly biased toward areas where active research and monitoring has taken place since 1990.

\section{Definitions}

In simplest terms, 'bycatch' refers to animals that become hooked, trapped, or entangled in fishing gear deployed with the intention of catching something else, i.e. the catching is inadvertent or accidental. However, it can be useful to distinguish between unintentional catch that is discarded (bycatch) and unintentional catch that is retained for consumption or sale (non-target catch) (Hall 1996, Read 2008). In most of the literature reviewed for this study, such a distinction was not made, and therefore we were able to do little more than flag it and accept that our compilation of bycatch data represents a mixture of both discarded and retained unintentional catches. It is also important to recognize that there is a large 'gray area' in some instances, where the demarcation between intentional and unintentional catch is 
blurred. There is no better example than Central Peru, where a major large-mesh driftnet fishery targeting both cetaceans (mainly dusky dolphins) and pelagic fish and elasmobranchs has operated since the 1980s (Read et al. 1988, Van Waerebeek \& Reyes 1994a,b). This 'directed' gillnet fishery accounted (and may still account) for a high proportion of the total landings of dusky dolphins and common dolphins Delphinus spp. in Peru, but partitioning catch estimates (based largely on observations at landing sites, market surveys, and data on fishing effort) between intentional and unintentional is problematic, at best.

Another aspect of bycatch that must be considered is what we call 'cryptic' bycatch, i.e. the animals that become entangled in fishing gear and either swim away injured, sometimes with gear still attached, and die even though they are not 'caught' or accounted for in bycatch statistics. Such events are an important component of the bycatch of large whales, but small cetaceans, pinnipeds, and sirenians also sometimes die in nets and drop out during haul-back, or escape with serious injuries. It is also worth remembering that bycatch occurs not only when gear is being actively fished, but also when it has been lost or abandoned, resulting in what is referred to as 'ghost fishing.'

Efforts have been made in both the United Nations Fisheries and Agriculture Organization (FAO) and the IWC to standardize terminology for fishing gear and practices. Here we adopt the FAO definitions, as used by the IWC Secretariat for compiling and coding cetacean bycatch data, such that 'gillnets' include: set gillnets (anchored), fixed gillnets (on stakes), driftnets, trammel nets, and various unspecified gill and 'entangling' nets. We also include, for the purposes of this paper, shark control nets and large-mesh predator exclusion nets associated with aquaculture facilities. An interesting variation of gillnetting occurs in southern Brazil, where a high proportion of the coastal gillnet vessels search for schools of bluefish Pomatomus saltatrix and 'run the net around the school (Secchi et al. 1997, p. 655).' Although in that sense the net is deployed like a purse seine, the bottom of this 'runaround' net is not pursed and therefore it functions as an actively fished gillnet. Similar use of gillnets occurs elsewhere, such as in the fishery for large croakers (Gulf corvina Cynoscion othonopterus) in the northern Gulf of California, Mexico, where this fishing method, however, does not appear to represent a bycatch threat to the Critically Endangered vaquita (Rojas-Bracho et al. 2006).

\section{Species list}

For taxonomy and nomenclature, we relied on the list of marine mammals maintained (and updated online) by the Society for Marine Mammalogy's Committee on Taxonomy (2012). This included, as of October 2012, 86 extant species of cetaceans, 32 pinnipeds, 4 sirenians, plus the 2 obligate marine otters (sea otter Enhyra lutris and marine otter Lontra felina). Many of these species have very extensive ranges, exhibiting considerable subspecies and population structure. For example, 20 cetacean species are subdivided into a total of 52 subspecies, and a total of 22 subspecies are recognized within 9 species of pinnipeds (Committee on Taxonomy 2012). In addition, 100s of geographically separate populations or stocks of marine mammals are recognized by treaty organizations (e.g. IWC), regional management bodies (e.g. North Atlantic Marine Mammal Commission, NAMMCO), and national agencies (e.g. US National Marine Fisheries Service and US Fish and Wildlife Service). In the USA alone, 28 stocks of common bottlenose dolphins Tursiops truncatus (Waring et al. 2011, Carretta et al. 2011) are currently subject to separate assessment and management under the Marine Mammal Protection Act. Also, NAMMCO has assessed and offered advice on 17 'aggregations' of narwhals Monodon monoceros and 25 of belugas Delphinapterus leucas in the North Atlantic and adjacent waters (NAMMCO 2000), while the IWC has identified no fewer than 9 additional beluga stocks elsewhere in that species' circumpolar range (IWC 2000). Ideally, a study such as the present one would be framed around a complete array of 'units to conserve' for all marine mammal species (Taylor 2005), but that ideal is far beyond our reach at present. Under the circumstances, we took a pragmatic approach similar to that used in the 1990 workshop report (IWC 1994), breaking down the species and subspecies into regional- or national-level units, often according to the availability of bycatch data.

\section{Sources of data on abundance (population size)}

For an initial baseline of abundance data, we used the most recently available documentation for the IUCN Red List of Threatened Species (http://www. iucnredlist.org/). All or nearly all of the species and many of the subspecies on the Society for Marine Mammalogy's list are included in the Red List documentation. However, that documentation is uneven 
in a number of respects, and therefore we made an effort to supplement, revise, and update the data by drawing on the literature (both published and 'gray') and by communicating directly with expert informants.

\section{Sources of information and data on bycatch}

To identify and obtain much of the primary literature, we used internet search engines (e.g. Google Scholar) and online university libraries, with keywords such as 'bycatch,' 'entanglement,' 'incidental catch,' 'catch,' marine mammals (e.g. 'pinniped'), fishing gear types (e.g. 'driftnet'), and species (e.g. 'short-beaked common dolphin' and 'Delphinus delphis') in various combinations. We also consulted extensively with regional and local experts on marine mammals and fishery bycatch, not only to identify and obtain relevant documents, but also to gain insights on information gaps and to verify or clarify provisional findings. Although we would have preferred to limit our search to the primary (peerreviewed) literature, we recognized that so-called gray literature is often a major source of credible bycatch information and data. Therefore, we included in our search government reports (e.g. US Department of Commerce NOAA/NMFS Stock Assessment Reports), reports of multilateral or international bodies (e.g. IWC, NAMMCO, Convention on Migratory Species), reports published by non-governmental organizations (e.g. World Wildlife Fund, International Fund for Animal Welfare, Whale \& Dolphin Conservation Society), and abstracts of conference proceedings (e.g. Society for Marine Mammalogy, European Cetacean Society, Latin American Society of Specialists in Aquatic Mammals). These sources were used cautiously in view of the tendency for facts and figures reported (for example, in an abstract 'published' in a conference proceedings document) to differ from those given in a journal paper that is eventually published on the same study. At the same time, however, it was recognized that much of the gray literature is subjected to extensive peer review before public release, such that, in some instances, it is at least as reliable as the primary literature.

\section{Structure and composition of database}

In several spreadsheets (Excel) we recorded the reported bycatch of all marine mammals in all types of gear and fisheries, worldwide, 1990 to 2011. For each species, subspecies, and subpopulation, we entered the most recent abundance estimate available and the reported or estimated bycatch by gear type, location (region, country, port), and year or period of years. We also noted in every case how the bycatch data had been obtained by the reporting source-e.g. numbers reported by onboard observers, in fishing vessel logbooks, or from interviews with fishermen; numbers observed at port landings, markets, or waste disposal sites; numbers observed stranded (or in some cases floating dead or injured at sea) and known or inferred to have been bycaught (from gear on the body or injuries consistent with fishing gear interaction). All numerical values were coded to indicate whether they represented actual counts (of observed individual animals or carcasses) or estimates (extrapolations from observations). When available from the source, we recorded the target species of the fishery in which the bycatch occurred.

As mentioned earlier, a problem that applies particularly to data on bycatch of large whales is that a high proportion of entangled animals escape, either by their own efforts or occasionally with the help of 'disentanglement' teams; in other words, many entanglements are non-lethal, at least initially (Knowlton \& Kraus 2001, Knowlton et al. 2008, Meÿer et al. 2011). It is generally agreed that determinations of how serious an injury is (i.e. how likely it is that the injury will prove lethal) should be made on a caseby-case basis (Andersen et al. 2008). In some instances, the data provided in the source have been pre-screened by experts, and thus can be taken at face value. For example, in the US Stock Assessment Reports, events (or 'incidents'; Meÿer et al. 2011) involving deaths and 'serious' injuries are reported as such, with an indication of whether entanglement was judged to be the 'primary' cause as well as a description of the nature of the evidence (e.g. gear on the body, characteristic wounds). This makes it possible to report a lower bound on the bycatch (deaths and serious injuries combined) by species/stock and by year, but such compilations take no account of the undetected, unreported (i.e. cryptic) component of bycatch. In other words, there is no clear way of actually estimating annual removals due to entanglement by species/stock in the absence of a systematic sampling program. In this study, we have tried to sort and annotate the large pool of whale bycatch data in a way that recognizes the underlying uncertainties, allowing us to at least qualify our conclusions appropriately. 


\section{Data management and analysis}

Because the focus of this study was gillnet bycatch (as defined above), we segregated the data so that an analysis similar to that presented in the 1990 workshop report (IWC 1994) could be carried out. Although the format of the 1990 report may not have been optimal, we chose to organize our data in a way that would facilitate comparisons with bycatch figures from that study as an historical benchmark. The bycatch data were sorted by species and by year and then assigned to geographical strata corresponding, to the extent possible, to those used previously (IWC 1994, their Table 1). In only a very few cases did we have a complete time series (1990 to 2011) of annual bycatch numbers for a species, subspecies, or population, or for a particular geographical area. There was great variability not only in the completeness of the data, but also in their nature and quality. In many instances, there was no way to judge how close the number of bycaught animals reported in a given document might be to the true number taken in that fishery or area that year. In the best cases, quantitative estimates with measures of uncertainty were provided, and the authors offered critical commentary on reliability and completeness. Much more often, however, the bycatch counts or estimates could only be interpreted as lower bounds because sampling was partial or the data were collected opportunistically. The difficulty we encountered trying to standardize and summarize the numerical catch data was not unexpected. In fact, it was consistent with that encountered at the 1990 workshop, where the vast majority of values for 'number killed per annum' were imprecise, e.g. some, $>$ a rounded figure, low 10s, 1000s, or <1 (IWC 1994, their Table 1). Instead of attempting to generate a single accurate and representative estimate of the annual bycatch in every case, we reported the range of numbers given by our sources for the entire period from 1990 to 2011.

The basic approach for generating entries in the 1990 to 2011 columns of our summary catch tables (see Tables 2 to 5) was as follows:

- When data were available for $>1 \mathrm{yr}$, we reported the range in annual counts or estimates for the years covered. For example, for the short-beaked common dolphin off the United States west coast, between 26 and 191 dolphins were estimated to be taken annually from 1997 to 2006.

- When data were available for only $1 \mathrm{yr}$, that number was reported along with the year.

- When bycatch was reported as a single number spanning >1 yr (e.g. 10 animals taken between 1990 and 1999, inclusive), we reported the number and the span of years.

- Given the inherent incompleteness and uncertainties associated with bycatch data, estimates based on a sample of direct observations are generally more credible than simple counts (total dead animals observed). In other words, we consider it more likely that simple reported counts be biased low than that estimates extrapolated from such counts will be biased high. In Tables 2 to 5, we reported both counts and estimates when they applied to different years, but if both a count and an estimate were available for a given year, we reported only the count in our table. - It was occasionally necessary to present values as upper or lower bounds only, i.e. as $>$ or $<$ a number, or to follow IWC (1994) by using terms such as some or low 10 s, etc.

The data given in the illustrative tables presented here were selected from the larger database (see the supplement at www.int-res.com/articles/suppl/n020 p071_supp.pdf), with the intention of making meaningful then-and-now comparisons, at least for cetaceans and pinnipeds, using as baselines the 1990 IWC workshop report (1994, their Table 1) and Woodley \& Lavigne (1991), respectively. We selected information for presentation in the tables with the goal of providing an overview of species and areas that are both data rich and data poor. It is important to emphasize that the information in all of our tables is as reported in the literature, and therefore a missing dimension is the expert opinion that formed the basis for many of the entries in IWC (1994, their Table 1). The known incompleteness of reporting throughout much of the world and the differences in methodology between the previous overviews and ours make almost any attempt at a then-and-now comparison problematic.

\section{RESULTS}

The database was compiled from $>900$ published sources and a few 'personal communications.' Around 570 of the sources contained information on gillnet bycatch. Numerical data on gillnet bycatch levels or rates were available from 90 countries or overseas territories. Gillnet bycatch included odontocetes in at least 73 countries, mysticetes in at least 28 , pinnipeds in 25, sirenians in 32, and mustelids in 3 (Table 1). It is important to emphasize that Table 1 reflects only what we could find on gillnet bycatch in the literature, supplemented by unpublished information from colleagues in a few instances. Unques- 
tionably, this table under-represents the true situation in that many more countries than indicated have probably experienced gillnet bycatch of a given taxonomic group even though such bycatch was undocumented or unreported in the literature that we examined.

There were relatively few complete time series of annual counts or estimates of gillnet bycatch for an entire species, subspecies, or lower unit (e.g. subpopulations or stocks) over its full geographic range. Frequently, the bycatch data, whether in the form of an estimate, a simple body count, or a mere statement that bycatch occurs, were presented in reference to a specific area or fishery rather than to a population of animals.

\section{Odontocete cetaceans}

Bycatch in gillnets continues to affect many odontocete species; 61 of 74 recognized species (82\%) have reportedly been bycaught in some kind of fishing gear somewhere in their range since 1990, and 56 species $(75 \%)$ have been bycaught in gillnets. Although, in many instances, it appears that bycatch counts or estimates have increased since 1990 (Table 2), we emphasize that this does not necessarily mean the actual scale of the bycatch has increased. In many instances it reflects, instead, changes in monitoring and reporting effort. Sri Lanka is one of the few countries with very high estimates of cetacean bycatch in the late 1980s and early 1990s, but very little new quantitative bycatch data since then (see 'Discussion'). In contrast, documentation of cetacean bycatch continued through the early 2000s in Peru, where very high bycatch levels had been documented in the 1980s and early 1990s and continue unabated (again, see 'Discussion'). We found few examples of reliable data on trends in gillnet bycatch rates (Table 2). Although fatal entanglements of odontocetes in aquaculture anti-predator nets appear to be infrequent, dolphin deaths in such nets have been reported from salmon and tuna facilities in Australia and Chile (Kemper et al. 2003).

Numbers of individuals killed in gillnets tend to be greatest for species that are widely distributed in coastal and shelf waters. Common dolphins and striped dolphins, for example, have continued to be taken in large numbers globally despite the fact that large-scale driftnet fishing on the high seas has been illegal since 1993, eliminating one source of very large bycatches of northern right whale dolphins and common dolphins. Although the conservation signif- icance of the large ongoing bycatches of common and striped dolphins is not entirely clear, there is reason for concern in some areas, certainly in Peru, Ecuador, and the Mediterranean, if not also in parts of the European Atlantic. With greatly improved bycatch monitoring, reported annual bycatches of these dolphins have been in the 1000s off western Europe. In the 1990s (post-1994) an illegal Spanish driftnet fleet for swordfish Xiphias gladius and sunfish Mola mola took 100s of common and striped dolphins in the western Mediterranean each year, and additional unknown (but probably large) numbers were taken by Italian and Moroccan driftnet vessels operating illegally in the region at the time (Silvani et al. 1999). Surface driftnet fleets from Ireland, France, and the UK targeting albacore tuna Thunnus alalunga in the Bay of Biscay-Celtic Sea region killed an estimated 11723 (7670 to 15776) common dolphins and 12635 (10009 to 15261 ) striped dolphins from 1990 to 2000 (Rogan \& Mackey 2007). Although those albacore fisheries had closed by 2002 (Rogan \& Mackey 2007), the large Moroccan driftnet fleet continued to operate in the Alborán Sea (southwestern Mediterranean) and in and around the Strait of Gibraltar, causing an estimated bycatch over a 12 mo period, based on onboard observer and fishing effort data from 2002 to 2003, of 3110 to 4184 and 11589 to 15127 dolphins (common and striped combined) in the 2 regions, respectively (Tudela et al. 2005). Driftnets were not the only sources of bycatch mortality for the common and striped dolphin populations: many 100s were also being taken (and continue to be taken) annually in set gillnets and trammel nets as well as trawl nets (e.g. Tregenza et al. 1997, Fernández-Contreras et al. 2010). In the South Pacific, the average estimated annual gillnet bycatch of longbeaked common dolphins by vessels from a single Peruvian port (Salaverry) from 2002 to 2007 was 973 (541 to 1550) (Mangel et al. 2010). Salaverry was estimated to host only about $2 \%$ of the total Peruvian gillnet fleet at the time. In fact, Mangel et al. (2010) speculated that the total annual mortality of small cetaceans in the Peruvian artisanal fishery during the first decade of the 21st century could have been as high as, or even higher than, the estimated 15000 to 20000 in the early 1990s (Van Waerebeek \& Reyes 1994b). A similar situation exists in Ecuador, where a very large artisanal gillnetting fleet has continued to operate, but with only limited bycatch monitoring (Félix \& Samaniego 1994, Félix et al. 2007).

Similarly, very large numbers of harbor porpoises continue to be taken in gill and trammel nets in the North Atlantic and its adjoining seas, even though 
Table 1. Bycatch in gillnets from 1990 to 2011 by country and/or territory. X means that we have at least 1 confirmed record of gillnet bycatch. Such bycatch almost certainly has occurred in many more countries, and for listed countries involved more taxa than shown here

\begin{tabular}{|c|c|c|c|c|c|c|c|c|c|c|c|}
\hline & $\begin{array}{l}\text { Odonto- } \\
\text { cetes }\end{array}$ & $\begin{array}{l}\text { Mysti- } \\
\text { cetes }\end{array}$ & $\begin{array}{l}\text { Pinni- } \\
\text { peds }\end{array}$ & Sirenians & $\begin{array}{l}\text { Muste- } \\
\text { lids }\end{array}$ & & $\begin{array}{l}\text { Odonto- } \\
\text { cetes }\end{array}$ & $\begin{array}{l}\text { Mysti- } \\
\text { cetes }\end{array}$ & $\begin{array}{l}\text { Pinni- } \\
\text { peds }\end{array}$ & Sirenians & $\begin{array}{l}\text { Muste- } \\
\text { lids }\end{array}$ \\
\hline Argentina & $\mathrm{X}$ & & & & & Malaysia & $\mathrm{X}$ & & & $\mathrm{X}$ & \\
\hline Australia & $\mathrm{X}$ & $\mathrm{X}$ & $\mathrm{X}$ & $\mathrm{X}$ & & Mauritania & $\mathrm{X}$ & & & $\mathrm{X}$ & \\
\hline Bangladesh & $\mathrm{X}$ & & & & & Mayotte & $\mathrm{X}$ & & & $\mathrm{X}$ & \\
\hline Belgium & $\mathrm{X}$ & & $\mathrm{X}$ & & & Mexico & $\mathrm{X}$ & $\mathrm{X}$ & & $\mathrm{X}$ & \\
\hline Belize & & & & $\mathrm{X}$ & & Montenegro & $\mathrm{X}$ & & & & \\
\hline Bolivia & $\mathrm{X}$ & & & & & Morocco & $\mathrm{X}$ & $\mathrm{X}$ & & & \\
\hline Brazil & $\mathrm{X}$ & $\mathrm{X}$ & $\mathrm{X}$ & $\mathrm{X}$ & & Mozambique & $\mathrm{X}$ & & & $\mathrm{X}$ & \\
\hline Bulgaria & $\mathrm{X}$ & & & & & Myanmar & $\mathrm{X}$ & & & $\mathrm{X}$ & \\
\hline Cambodia & $\mathrm{X}$ & & & $\mathrm{X}$ & & New Zealand & $\mathrm{X}$ & $\mathrm{X}$ & $\mathrm{X}$ & & \\
\hline Cameroon & & & & $\mathrm{X}$ & & Nigeria & & & & $\mathrm{X}$ & \\
\hline Canada & $\mathrm{X}$ & $\mathrm{X}$ & $\mathrm{X}$ & & & Norway & $\mathrm{X}$ & & $\mathrm{X}$ & & \\
\hline Chile & $\mathrm{X}$ & $\mathrm{X}$ & & & & Oman & $\mathrm{X}$ & $\mathrm{X}$ & & & \\
\hline China & $\mathrm{X}$ & $\mathrm{X}$ & & & & Peru & $\mathrm{X}$ & $\mathrm{X}$ & $\mathrm{X}$ & & $\mathrm{X}$ \\
\hline Colombia & $\mathrm{X}$ & $\mathrm{X}$ & $\mathrm{X}$ & $\mathrm{X}$ & & Philippines & $\mathrm{X}$ & & & & \\
\hline Congo & $\mathrm{X}$ & & & & & Poland & $\mathrm{X}$ & & $\mathrm{X}$ & & \\
\hline Croatia & $\mathrm{X}$ & & & & & Portugal & $\mathrm{X}$ & & & & \\
\hline Dagestan & & & $\mathrm{X}$ & & & Puerto Rico & $\mathrm{X}$ & & & $\mathrm{X}$ & \\
\hline Denmark & $\mathrm{X}$ & & $\mathrm{X}$ & & & Rep. of Congo & & & & $\mathrm{X}$ & \\
\hline Ecuador & $\mathrm{X}$ & $\mathrm{X}$ & & & & Romania & $\mathrm{X}$ & & & & \\
\hline Faroe Islands & $\mathrm{X}$ & & & & & Russia & $\mathrm{X}$ & $\mathrm{X}$ & $\mathrm{X}$ & & \\
\hline Finland & & & $\mathrm{X}$ & & & Sierra Leone & $\mathrm{X}$ & & & $\mathrm{X}$ & \\
\hline France & $\mathrm{X}$ & $\mathrm{X}$ & $\mathrm{X}$ & & & Singapore & & & & $\mathrm{X}$ & \\
\hline French Guiana & & & & $\mathrm{X}$ & & Slovenia & $\mathrm{X}$ & & & & \\
\hline Gabon & & & & $\mathrm{X}$ & & South Africa & $\mathrm{X}$ & $\mathrm{X}$ & $\mathrm{X}$ & & \\
\hline Georgia & $\mathrm{X}$ & & & & & South Korea & $\mathrm{X}$ & $\mathrm{X}$ & & & \\
\hline Germany & $\mathrm{X}$ & & & & & Spain & $\mathrm{X}$ & & & & \\
\hline Ghana & $\mathrm{X}$ & & & & & Sri Lanka & $\mathrm{X}$ & & & $\mathrm{X}$ & \\
\hline Greece & $\mathrm{X}$ & & $\mathrm{X}$ & & & Sweden & $\mathrm{X}$ & & $\mathrm{X}$ & & \\
\hline Greenland & $\mathrm{X}$ & $\mathrm{X}$ & & & & Taiwan & $\mathrm{X}$ & & & & \\
\hline Guinea & & & & $\mathrm{X}$ & & Tanzania & $\mathrm{X}$ & $\mathrm{X}$ & & $\mathrm{X}$ & \\
\hline Guinea-Bissau & & & & $\mathrm{X}$ & & Thailand & $\mathrm{X}$ & & & $\mathrm{X}$ & \\
\hline Hong Kong & $\mathrm{X}$ & & & & & The Netherlands & $\mathrm{X}$ & & & & \\
\hline Iceland & $\mathrm{X}$ & $\mathrm{X}$ & $\mathrm{X}$ & & & Togo & & $\mathrm{X}$ & & & \\
\hline India & $\mathrm{X}$ & & & $\mathrm{X}$ & & Tunisia & $\mathrm{X}$ & & & & \\
\hline Indonesia & $\mathrm{X}$ & $\mathrm{X}$ & & & & Turkey & $\mathrm{X}$ & & $\mathrm{X}$ & & \\
\hline Iran & $\mathrm{X}$ & & $\mathrm{X}$ & & & Turkmenistan & & & $\mathrm{X}$ & & \\
\hline Ireland & $\mathrm{X}$ & $\mathrm{X}$ & $\mathrm{X}$ & & & Ukraine & $\mathrm{X}$ & & & & \\
\hline Israel & $\mathrm{X}$ & $\mathrm{X}$ & & & & Union of the & & & & $\mathrm{X}$ & \\
\hline Italy & $\mathrm{X}$ & $\mathrm{X}$ & & & & Comoros & & & & & \\
\hline Japan & $\mathrm{X}$ & $\mathrm{X}$ & $\mathrm{X}$ & $\mathrm{X}$ & $\mathrm{X}$ & United Arabian & & & & $\mathrm{X}$ & \\
\hline Kazakhstan & & & $\mathrm{X}$ & & & Emirates & & & & & \\
\hline Kenya & $\mathrm{X}$ & & & $\mathrm{X}$ & & United Kingdom & $\mathrm{X}$ & $\mathrm{X}$ & & & \\
\hline Laos & $\mathrm{X}$ & & & & & USA & $\mathrm{X}$ & $\mathrm{X}$ & $\mathrm{X}$ & $\mathrm{X}$ & $\mathrm{X}$ \\
\hline Liberia & & & & $\mathrm{X}$ & & Uruguay & $\mathrm{X}$ & & & & \\
\hline Lithuania & $\mathrm{X}$ & & & & & Venezuela & $\mathrm{X}$ & & & $\mathrm{X}$ & \\
\hline Madagascar & $\mathrm{X}$ & $\mathrm{X}$ & & & & Vietnam & $\mathrm{X}$ & & & & \\
\hline
\end{tabular}

the collapse and closure of many groundfish fisheries (e.g. for Atlantic cod Gadus morhua) beginning in the early 1990s, together with implementation of season or area closures and mandatory pinger programs in the 1990s and first decade of the 21st century, substantially reduced the levels of porpoise bycatch in some areas. In a thorough review, Stenson (2003) summarized bycatch estimates in all areas (available through about 2002), ranging as high as 2100 (CV = 0.18) in the New England sink gillnet fishery in 1994; $572(\mathrm{CV}=0.35)$ in the United States 'mid-Atlantic' coastal gillnet fishery in 1997; $474(\mathrm{SE}=224)$ in Canada's sink gillnet fishery in the Bay of Fundy in 1993; $>2000$ in various gillnet fisheries in Newfoundland in 
Table 2. Illustrative examples of reported odontocete bycatch mortality in gillnets before 1990 (from IWC 1994) and from 1990 to 2010 (our database; see 'Results' and Table S1 in the supplement at www.int-res.com/articles/suppl/n020p071_supp.pdf). Pre-1990 numbers are annual estimates determined by 1990 International Whaling Commission (IWC) workshop participants. Numbers for 1990 to 2010 are generally not annual, but rather counts or estimates (as given in the source documents) for 1 yr (single number), totals over a range of years $\left({ }^{\mathrm{a}}\right)$, or a range of annual numbers over a range of years $\left({ }^{b}:\right.$ in some instances with a count for the low end and an estimate for the high end; thus, a number and dash appear in the Count column and a number with no punctuation in the Estimate column). Source numbers refer to the 'Literature Cited' in the supplement. Note that numbers are as reported in the literature and, therefore, for most species and areas, they are negatively biased to an unknown but often probably large degree. NA: not applicable; not addressed by the 1990 workshop. Unk.: unknown; no information available. FMA: Franciscana Management Area

\begin{tabular}{|c|c|c|c|c|c|c|}
\hline Species & Location & Pre-1990 & $\begin{array}{l}1990- \\
\text { Count }\end{array}$ & $\begin{array}{l}-2010 \\
\text { Estimate }\end{array}$ & Years/Notes & Sources \\
\hline \multicolumn{7}{|l|}{ Delphinidae } \\
\hline \multirow[t]{5}{*}{ Delphinus delphis } & Australia & $>1000$ & $1-15^{\mathrm{b}}$ & & $1990-2009$ & $\begin{array}{c}25,26,28,103,215,218,228,230,232 \\
288,420,425,426,429,430,538\end{array}$ \\
\hline & Ecuador & NA & & 1118 & 1993 & 198 \\
\hline & Northwest Atlantic & $211-422$ & & $11-893^{b}$ & $1990-2009$ & $\begin{array}{l}72,261,401,518,520,543-547,549- \\
551,553,554\end{array}$ \\
\hline & European Atlantic & Some & $1-$ & $2522^{\mathrm{b}}$ & $1990-2009$ & $\begin{array}{l}51,86,87,131,190,227,251,260,262 \\
263,321,323,386-388,390,447,456 \\
457,459,461,470,485,486,496,497 \\
509,511\end{array}$ \\
\hline & $\begin{array}{l}\text { Strait of } \\
\text { Gibraltar }\end{array}$ & NA & & $>10000$ & $\begin{array}{l}\text { 2003; combined } \\
\text { short-beaked } \\
\text { common and } \\
\text { striped dolphins }\end{array}$ & 260,516 \\
\hline $\begin{array}{l}\text { Delphinus delphis } \\
\text { ponticus }\end{array}$ & Black Sea & NA & $1-297^{b}$ & & $1968-2007$ & $3,94,96,391,414,433$ \\
\hline $\begin{array}{l}\text { Delphinus } \\
\text { capensis }\end{array}$ & $\begin{array}{l}\text { Peru } \\
\text { South Africa }\end{array}$ & $\begin{array}{c}<50 \\
33\end{array}$ & $\begin{array}{c}7- \\
10-40^{b}\end{array}$ & $1186^{\mathrm{b}}$ & $\begin{array}{l}1991-2008 \\
2001-2005\end{array}$ & $\begin{array}{l}9,344,525,530,531 \\
395-399\end{array}$ \\
\hline $\begin{array}{l}\text { Lagenodelphis } \\
\text { hosei }\end{array}$ & $\begin{array}{l}\text { West Pacific, } \\
\text { Indian Ocean, and } \\
\text { Eastern Atlantic }\end{array}$ & Some & $1-$ & $31^{\mathrm{b}}$ & $1991-2005$ & $18,153,172,177,188,393,540,555$ \\
\hline $\begin{array}{l}\text { Lagenorhynchus } \\
\text { albirostris }\end{array}$ & North Atlantic & Some & $1-$ & $15^{\mathrm{b}}$ & $1990-2007$ & $205,377,378,380,381,382$ \\
\hline $\begin{array}{l}\text { Lagenorhynchus } \\
\text { acutus }\end{array}$ & North Atlantic & $<5$ & $1-$ & $240^{\mathrm{b}}$ & $\begin{array}{l}\text { 1990-2008; } \\
\text { mostly western }\end{array}$ & $\begin{array}{l}72,205,251,401,461,497,503,520 \\
541-545,548-552,554\end{array}$ \\
\hline $\begin{array}{l}\text { Lagenorhynchus } \\
\text { obliquidens }\end{array}$ & North Pacific & 11000 & $1-$ & $4519^{b}$ & $1990-2006$ & $\begin{array}{l}22,23,39,68,69,123-125,208,255 \\
368,369,491,498-501,559\end{array}$ \\
\hline $\begin{array}{l}\text { Lagenorhynchus } \\
\text { obscurus }\end{array}$ & Peru & $>1800$ & $2-$ & $1284^{\mathrm{b}}$ & $1990-2008$ & $343,345,525,530,531$ \\
\hline $\begin{array}{l}\text { Lagenorhynchus } \\
\text { australis }\end{array}$ & South America & Low $10 \mathrm{~s}$ & Some & & 1994-1997 & 224,325 \\
\hline $\begin{array}{l}\text { Lagenorhynchus } \\
\text { cruciger }\end{array}$ & All & NA & Unk. & & & \\
\hline Stenella attenuata & Ghana & NA & $1-14^{\mathrm{b}}$ & & 1998-2004 & 177,393 \\
\hline Stenella frontalis & Brazil & Some & $<10^{\mathrm{b}}$ & & $1990-2005$ & $182,183,348,422,567$ \\
\hline $\begin{array}{l}\text { Stenella } \\
\text { longirostris }\end{array}$ & $\begin{array}{l}\text { Zanzibar } \\
\text { Southwest Pacific }\end{array}$ & $\begin{array}{l}\text { NA } \\
>1000\end{array}$ & $\begin{array}{c}1 \text { to }>50^{\mathrm{b}} \\
5^{\mathrm{a}}\end{array}$ & & $\begin{array}{l}1995-2008 \\
1999-2009\end{array}$ & $\begin{array}{l}15-17,448,449 \\
65,103,216-218,228,232,233\end{array}$ \\
\hline Stenella clymene & Ghana & NA & $1-31^{b}$ & & $1998-2008$ & 177,393 \\
\hline \multirow[t]{3}{*}{$\begin{array}{l}\text { Stenella } \\
\text { coeruleoalba }\end{array}$} & Northeast Atlantic & Some & $1-$ & $1793^{\mathrm{b}}$ & $1990-2008$ & $\begin{array}{l}51,87,131,190,227,251,262,263,461 \\
\quad 470,496\end{array}$ \\
\hline & Strait of Gibraltar & NA & & $>10000$ & $\begin{array}{l}\text { 2003; combined } \\
\text { short-beaked } \\
\text { common and } \\
\text { striped dolphins }\end{array}$ & 516 \\
\hline & $\begin{array}{l}\text { Mediterranean } \\
\text { Sea }\end{array}$ & $5000-10000$ & $1-$ & $1800^{\mathrm{b}}$ & 1990-2008 & $\begin{array}{r}2,64,102,104,132-144,184-186,192 \\
209,210,260,261,268,269,289,321 \\
347,357,366,407,411,481,486,517\end{array}$ \\
\hline
\end{tabular}


Table 2 (continued)

\begin{tabular}{|c|c|c|c|c|c|c|}
\hline Species & Location & Pre-1990 & $\begin{array}{l}1990- \\
\text { Count }\end{array}$ & $\begin{array}{l}\text {-2010 } \\
\text { Estimate }\end{array}$ & Years/Notes & Sources \\
\hline Tursiops truncatus & $\begin{array}{l}\text { US East Coast } \\
\text { Mediterranean } \\
\text { Sea }\end{array}$ & $\begin{array}{c}\text { Some } \\
110-455\end{array}$ & $1-$ & $\begin{array}{c}14-340^{\mathrm{b}} \\
35^{\mathrm{b}}\end{array}$ & $\begin{array}{l}1990-2008 \\
1991-2008\end{array}$ & $\begin{array}{l}72,401,518,520,541-545,551-553 \\
2,132-144,185,209,260,261,289,338 \\
\quad 407,481\end{array}$ \\
\hline Tursiops truncatus & Southwest Pacific & $>1700$ & $10^{\mathrm{a}}$ & & $1993-2007$ & $\begin{array}{l}27,28,103,215,216,228,232,288,425 \\
\quad 429,430\end{array}$ \\
\hline $\begin{array}{l}\text { Tursiops truncatus } \\
\text { ponticus }\end{array}$ & Black Sea & NA & $1-$ & $1500^{\mathrm{b}}$ & 1990-2009 & $\begin{array}{l}1,3,94,96,223,261,391,406,414,433 \\
507\end{array}$ \\
\hline Tursiops aduncus & Southeast Africa & NA & $22-50^{\mathrm{b}}$ & & $1995-2008$ & $15-19,292,373,395-399,439,440$ \\
\hline $\begin{array}{l}\text { Cephalorhynchus } \\
\text { commersonii }\end{array}$ & South America & Some & $1-$ & $179^{\mathrm{b}}$ & 1993-2009 & $\begin{array}{l}\text { 85, 224, 226, 270, 474, 478, 479, } \\
\text { R. N. P. Goodall pers. comm. (2011) }\end{array}$ \\
\hline $\begin{array}{l}\text { Cephalorhynchus } \\
\text { eutropia }\end{array}$ & All (Chile) & Some & $116^{\mathrm{a}}$ & & 1989-1991 & $106,171,400,437,444$ \\
\hline $\begin{array}{l}\text { Cephalorhynchus } \\
\text { heavisidii }\end{array}$ & $\begin{array}{l}\text { All (southwestern } \\
\text { Africa) }\end{array}$ & Some & Unk. & & & \\
\hline $\begin{array}{l}\text { Cephalorhynchus } \\
\text { hectori }\end{array}$ & All (New Zealand) & $27-95$ & $1-20^{\mathrm{b}}$ & & 1990-2009 & $60,130,146-152,191$ \\
\hline $\begin{array}{l}\text { Cephalorhynchus } \\
\text { hectori maui }\end{array}$ & $\begin{array}{l}\text { All (North Island, } \\
\text { New Zealand) }\end{array}$ & NA & $>10^{\mathrm{a}}$ & & $2001-2003$ & $146-148,171$ \\
\hline $\begin{array}{l}\text { Lissodelphis } \\
\text { borealis }\end{array}$ & $\begin{array}{l}\text { Northwest Pacific } \\
\text { US West Coast }\end{array}$ & 19000 & & $\begin{array}{l}000-19000^{\mathrm{b}} \\
5-71^{\mathrm{b}}\end{array}$ & $\begin{array}{l}1990-1994 \\
1991-2008\end{array}$ & $\begin{array}{l}368-370,505 \\
68,69,122-124,207\end{array}$ \\
\hline $\begin{array}{l}\text { Lissodelphis } \\
\text { peronii }\end{array}$ & Peru & $>5$ & $1^{\mathrm{a}}$ & & 1990 & 445 \\
\hline Feresa attenuata & Sri Lanka & $>170$ & $50^{\mathrm{a}}$ & & 1991-1992 & 172 \\
\hline Globicephala melas & Mediterranean Sea & $50-100$ & $1-$ & $132^{\mathrm{b}}$ & $1986-2003$ & $64,131-144,189$ \\
\hline $\begin{array}{l}\text { Globicephala } \\
\text { macrorhynchus }\end{array}$ & Indian Ocean & $>100$ & $<1^{\mathrm{b}}$ & & -2008 & 292 \\
\hline Grampus griseus & $\begin{array}{l}\text { Mediterranean Sea } \\
\text { Zanzibar }\end{array}$ & $\begin{array}{l}30-100 \\
\text { NA }\end{array}$ & $\begin{array}{l}1- \\
<20^{\mathrm{a}}\end{array}$ & $79^{\mathrm{b}}$ & $\begin{array}{l}1990-2003 \\
2000-2006\end{array}$ & $\begin{array}{l}132-144,185,261,407,411,481,517 \\
17,18,440,441\end{array}$ \\
\hline $\begin{array}{l}\text { Orcaella } \\
\text { brevirostris }\end{array}$ & $\begin{array}{l}\text { All (Bangladesh, } \\
\text { Mahakam River, } \\
\text { Malampaya Sound, } \\
\text { Mekong River, and } \\
\text { Songkhla Lake) }\end{array}$ & Some & $1-32^{\mathrm{b}}$ & & 1990-2007 & $\begin{array}{l}57,58,73,75,76,178,273,303,304 \\
346,436,492,493,495\end{array}$ \\
\hline Orcaella heinsohni & Australia & NA & $<5^{\mathrm{b}}$ & & $1993-2007$ & $28,228,230,232,233,245,425,426$ \\
\hline Orcinus orca & $\begin{array}{l}\text { All (global } \\
\text { distribution) }\end{array}$ & Some & $<1^{\mathrm{b}}$ & & 1993-2004 & $33,118,189,393,498,501$ \\
\hline $\begin{array}{l}\text { Peponocephala } \\
\text { electra }\end{array}$ & All (circumtropical) & $<10$ & $1-73$ & & 1992-2008 & $172,177,188,292,348,393$ \\
\hline $\begin{array}{l}\text { Pseudorca } \\
\text { crassidens }\end{array}$ & Sri Lanka & $>125$ & 33 & & 1991-1992 & 172 \\
\hline Sousa teuszii & West Africa & NA & Probable & & 2008 & 305,556 \\
\hline Sousa chinensis & Zanzibar & NA & $1-5^{b}$ & & 1995-2008 & $15-18,439-441$ \\
\hline Sotalia fluviatilis & $\begin{array}{l}\text { All (Amazon } \\
\text { River basin) }\end{array}$ & $>90$ & $<2^{\mathrm{b}}$ & & $1996-2007$ & $412,422,451$ \\
\hline Sotalia guianensis & $\begin{array}{l}\text { All (coastal marine } \\
\text { in northern South } \\
\text { America and } \\
\text { Central America) }\end{array}$ & NA & $3-$ & $115^{\mathrm{b}}$ & 2003-2009 & $\begin{array}{l}30,56,77,98,160,165-167,174,182 \\
183,193,202,204,350,359,422,423 \\
451,567\end{array}$ \\
\hline Steno bredanensis & $\begin{array}{l}\text { South Atlantic } \\
\text { (Ghana, Brazil) }\end{array}$ & Some & $1-6^{b}$ & & $1990-2010$ & $166-168,177,183,359,393,422,560$ \\
\hline
\end{tabular}


Table 2 (continued)

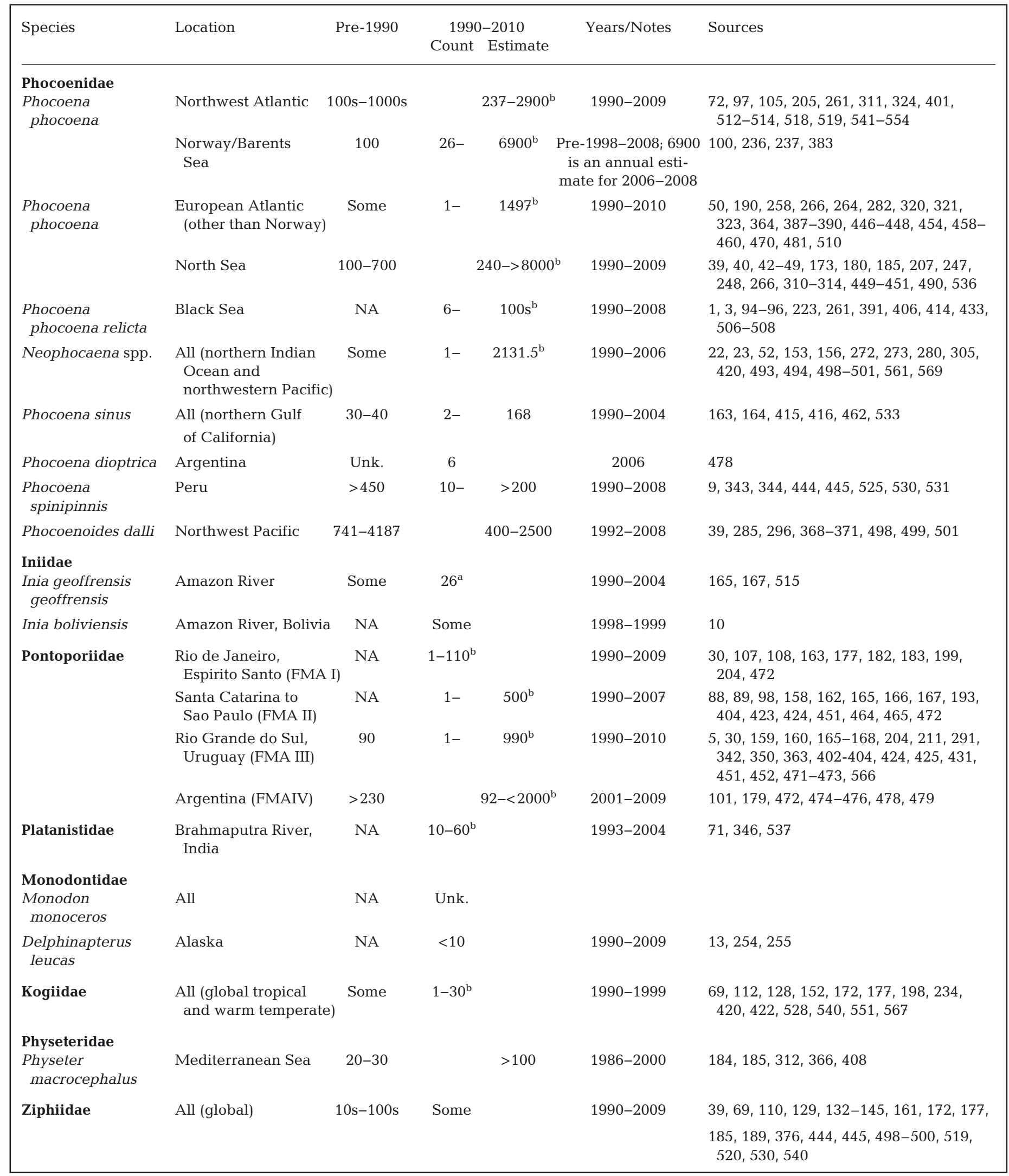


1992; 7366 in Danish and 818 (95\% CI = 674 to 1233) in UK fisheries for various bottom- and groundfish in the North Sea in 1994 and 1995, respectively; 2200 (95\% CI $=900$ to 3500 ) in hake gillnet and tangle and wreck net fisheries in the Celtic Sea in 1993; and 209 (95\% CI $=95$ to 475 ) in UK gillnet and tangle net fisheries for elasmobranchs and crayfish west of Scotland in 1997. Those numbers (together with other data summarized by Stenson) suggest that the total annual bycatch of harbor porpoises in gillnets in the North Atlantic was $>15000$ in the 1990s. Since the publication of Stenson's review, estimates have become available from the St. Lawrence River and Gulf of St. Lawrence $(2394,95 \%$ CI $=1440$ to 3348 in 2001: Lesage et al. 2006), Newfoundland (around 2200 in 2003: Benjamins et al. 2007), Iceland (1049, $95 \% \mathrm{CI}=$ 505 to 1599 in 2003: Ólafsdóttir 2009), and Norway (6900 in coastal fisheries for anglerfish Lophius piscatorius and cod in 2006 to 2008: Bjørge et al. 2011).

As in the case of common and striped dolphins, the overall conservation significance of the ongoing large bycatch of harbor porpoises in the North Atlantic is not clear, but the implications of continued gillnet bycatch for at least 2 populations are a major concern. More than 40 harbor porpoises from the critically endangered Baltic Sea population were caught in Polish waters alone, in either surface driftnets for salmonids or bottom-set gillnets for cod, flounder, and pike-perch, between 1990 and 1999 (Skóra \& Kuklik 2003). The estimated annual bycatch of harbor porpoises in German Baltic waters was 82 (presumably almost entirely in gillnets or other entangling gear) based on data collected between 1996 and 2002, leading Scheidat et al. (2008) to conclude that bycatch is a 'major threat' to porpoises throughout the western Baltic.

Bycatch, mainly in bottom-set gillnets for turbot Psetta maeotica, spiny dogfish Squalus acanthias, and sturgeon Acipenser spp., continues to be regarded as 'the most serious threat' to the Endangered Black Sea harbor porpoise subspecies Phocoena phocoena relicta (Birkun \& Frantzis 2008). The scale of this bycatch is thought to be at least in the 1000s annually, and it occurs in the territorial waters of all 6 riparian countries (Bulgaria, Georgia, Romania, Russia, Turkey, and Ukraine), largely in fisheries that are illegal, unreported, and/or unregulated (Birkun \& Frantzis 2008). In Turkey, some of the equipment and facilities used to process cetacean carcasses prior to the 1983 hunting ban was still being used in the 1990s to produce oil from cetaceans, especially harbour porpoises, bycaught in bottom-set gillnets (Tonay \& Öztürk 2012).
For many species of small odontocetes, some of which are of great conservation concern, the bycatch data presented here and in our database are misleading simply because no reliable quantitative documentation is available. This was true before 1990 and remains true today. For example, the 1990 IWC workshop report refers only to the fact that 'some' bycatch of Irrawaddy dolphins Orcaella brevirostris was known to occur in India and the northern Indian Ocean and that Australian snubfin dolphins O. heinsohni were killed to an uncertain extent in anti-shark nets off Queensland. Our post-1990 literature search revealed total documented catches of only a few individuals of both species per year. Yet since 1990, 5 very small subpopulations of $O$. brevirostris have been red listed as 'Critically Endangered' and the 2 species of Orcaella have been red listed as 'Vulnerable' (O. brevirostris) (Reeves et al. 2008a) and 'Near Threatened' (O. heinsohni) (Reeves et al. 2008b). In the Red List documentation for all of these subpopulations and both species, gillnet bycatch is identified as a major ongoing threat.

Similarly, there was virtually no numerical data on bycatch of endangered South Asian river dolphins Platanista gangetica in 1990, and that continues to be the case. As summarized in the Red List documentation (Smith \& Braulik 2008):

\begin{abstract}
Mortality in fishing gear, especially gillnets, is a severe problem for Ganges (South Asian river) dolphins throughout most of their range. They are particularly vulnerable because their preferred habitat is often in the same location as the fishing grounds. A specific problem in parts of India and Bangladesh is that, because dolphin oil is highly valued as a fish attractant, fishermen have a strong incentive to kill any animals found alive in their nets and even to set their nets strategically in the hope of capturing dolphins.
\end{abstract}

In spite of the concern,

Meaningful quantitative data on the magnitude of catches, either deliberate or incidental, are unavailable and unlikely to become available in the absence of a well-organized, adequately funded, and incorruptible fishery/wildlife management system (Smith \& Braulik 2008).

Another group of small cetaceans for which there is concern about the impacts of gillnet bycatch is the genus Cephalorhynchus, which consists of 4 species, all of them coastal endemics. The endangered New Zealand species, Hector's dolphin C. hectori, was considered at high risk in 1990, mainly because of annual gillnet bycatch levels (27 to 95) described as 'maybe not sustainable' (IWC 1994). Even though the available estimated and reported bycatch statistics since 1990 (Table 2) could be interpreted as implying 
that levels have declined, at least in part as a result of management efforts (protected area designation, use of pingers by some fishermen), this cannot be confirmed, and levels may still be unsustainable (Dawson \& Slooten 2005, Slooten 2007). The Chilean dolphin C. eutropia is red listed as 'Near Threatened', with bycatch in artisanal gillnets (Reyes \& Oporto 1994, Goodall et al. 1994, Bravo et al. 2010) and pinniped control nets set near salmon farms considered the principal threat (Reeves et al. 2008c). Commerson's dolphins C. commersonii, consisting of a southern South American subspecies C. C. Commersonii and an Indian Ocean subspecies C. c. kerguelenensis, may be more widely distributed and more abundant than the other species of the genus, but substantial bycatch occurs in artisanal gill and trammel nets as well as trawls (Reeves et al. 2008d). In one small part of Commerson's dolphins' range in Santa Cruz Province, Argentina, gillnet mortality in the fishing season 1999 to 2000 was nearly 180 dolphins (Iñíguez et al. 2003). Unlike Hector's dolphins, very little is known about abundance of Chilean and Commerson's dolphins, and therefore it would be difficult to assess the actual degree to which they are threatened by gillnet bycatch even if reliable data on bycatch levels were available.

Another regional endemic, the franciscana Pontoporia blainvillei in Brazil, Uruguay, and Argentina, has long been recognized as a bycatch concern, and bycatch levels, almost entirely in coastal gillnets (although franciscanas are also killed in shrimp trawls), continue to be very high in absolute terms. At a rangewide workshop in 2000 (Ott et al. 2002), experts reviewed the bycatch estimates, which could total up to nearly 2600 franciscanas $\mathrm{yr}^{-1}$ (sum of maximum values for all areas in their Table 1), but certainly total at least many 100s per year (sum of minimum values in their Table 1). Since the early 2000s, important progress has been made toward obtaining abundance estimates for some of the affected franciscana stocks (e.g. Secchi et al. 2001, Crespo et al. 2010).

The vaquita is generally considered the cetacean most likely to become extinct unless extreme measures are taken quickly to eliminate the risk of bycatch. The 1990 IWC workshop report indicated that catches in passive gear had been 32 to $33 \mathrm{yr}^{-1}$ in 1985 and 1990, based on 'direct counts' and that at least 7 more vaquitas had died in shrimp trawls since 1985 (IWC 1994). The first (and only) 'properly designed' study of vaquita bycatch took place between January 1993 and January 1995 using a combination of onboard observer and interview data
(Rojas-Bracho et al. 2006). The resulting estimate of $39 \mathrm{yr}^{-1}$ continues to be used as the 'best' estimate (D'Agrosa et al. 1995, 2000) in spite of the considerable changes that have taken place in fishing effort and management.

Entanglement in gillnets and trammel nets is regarded as 'a serious direct threat' to a small $(<100)$, Critically Endangered population of Indo-Pacific humpback dolphins Sousa chinensis in western Taiwan, even though the evidence is almost all circumstantial or indirect: 1000 s of these nets overlap with the dolphins' habitat and $>30 \%$ of photo-identified individuals bear scars or injuries 'most likely caused by interactions with fisheries' (Dungan et al. 2011). Similar concerns apply to other populations of humpback dolphins, finless porpoises Neophocaena spp., and Irrawaddy and Australian snubfin dolphins in Asia, Oceania, and Africa, where bycatch (and population) data are fragmentary, at best (Reeves et al. 1997, Jefferson 2004, Perrin et al. 2005, Reeves \& Wang 2011, Wang \& Reeves 2011). In some areas, such as Madagascar (Razafindrakoto et al. 2004), West Africa (Van Waerebeek et al. 2004), the Philippines (Dolar et al. 1994), Sri Lanka (Leatherwood \& Reeves 1989), and Oman (Baldwin et al. 2004), bycatch data are confounded by the fact that small cetaceans are taken deliberately for food and those caught incidentally are often eaten.

The beaked whales (Ziphiidae) have attracted much attention in recent years because of their susceptibility to ill effects from exposure to naval sonar (e.g. Simmonds \& Lopez-Jurado 1991, Frantzis 1998, Tyack et al. 2011). They are also vulnerable to gillnet entanglement, although in the data from 1990 onward reviewed here the numbers do not appear large (Table 2; Table S1) in the supplement). Of great interest is the apparently dramatic reduction in the bycatch rate of beaked whales in the California drift gillnet fishery after the introduction of pingers as a mitigation tool (Carretta et al. 2008, Carretta \& Barlow 2011). During the first 6 yr of an observer program (1990 to 1995), 33 beaked whales were observed bycaught in 3303 fishing sets, whereas from 1996 through 2006, not a single bycatch of a beaked whales was observed in 4381 sets.

\section{Mysticete cetaceans}

We found records indicating that at least 13 of the 14 recognized mysticete species were bycaught between 1990 and 2011; of those, 9 species are known to have been taken in gillnets (Table 3, 
Table 3. As in Table 2 but for illustrative mysticete bycatch. For all further details see Table 2 legend

\begin{tabular}{|c|c|c|c|c|c|}
\hline Species & Location & Pre-1990 & $\begin{array}{c}1990-2010 \\
\text { Count }\end{array}$ & Years/Notes & Sources \\
\hline \multicolumn{6}{|l|}{ Balaenidae } \\
\hline Balaena mysticetus & All (Arctic and sub-Arctic) & NA & Unk. & & \\
\hline Eubalaena glacialis & All (Northwest Atlantic) & $<1$ & $<4^{\mathrm{b}}$ & 1990-2006 & $72,219,275,326,544,554$ \\
\hline Eubalaena japonica & All (North Pacific) & $<1$ & Unk. & & \\
\hline \multirow[t]{3}{*}{ Eubalaena australis } & Australia & NA & 1,3 & 1993,1995 & 27,426 \\
\hline & South Africa & $<1$ & 1 & 2000 & 352 \\
\hline & Brazil & NA & 1,6 & 2003,2010 & 167,427 \\
\hline \multicolumn{6}{|l|}{ Balaenopteridae } \\
\hline \multirow[t]{2}{*}{ Balaenoptera physalus } & European Atlantic & NA & 1 & 1992,1995 & 131,449 \\
\hline & Mediterranean Sea & $<1$ & $<1^{\mathrm{b}}$ & 1986-1999 & 185,392 \\
\hline Balaenoptera borealis & $\begin{array}{l}\text { All (North Atlantic, North } \\
\text { Pacific, and Southern } \\
\text { Hemisphere) }\end{array}$ & NA & Unk. & & \\
\hline \multirow[t]{2}{*}{ Balaenoptera edeni } & Brazil & $<1$ & 1 & 2005 & 193 \\
\hline & Oman & NA & 1 & 2000,2001 & 356 \\
\hline Balaenoptera omurai & $\begin{array}{l}\text { All (southern Japan to } \\
\text { Indonesia and Papua } \\
\text { New Guinea) }\end{array}$ & NA & Unk. & & \\
\hline Balaenoptera musculus & All (global except Arctic) & NA & Unk. & & \\
\hline \multirow{2}{*}{$\begin{array}{l}\text { Balaenoptera } \\
\text { acutorostrata }\end{array}$} & Chile & NA & 1 & 2004 & 180 \\
\hline & Peru & NA & 1 & 1991,1993 & $385,445,529$ \\
\hline \multirow{2}{*}{$\begin{array}{l}\text { Balaenoptera } \\
\text { acutorostrata } \\
\text { acutorostrata }\end{array}$} & Mediterranean Sea & $<4$ & $<1^{\mathrm{b}}$ & 1998-2004 & $450,468,516$ \\
\hline & Northwest Atlantic & $10-20$ & $1-10^{\mathrm{b}}$ & 1990-2009 & $\begin{array}{l}66,72,205,277,278,313,315 \\
313,315,316,318,329,331- \\
333,384,520,545,551,554,555\end{array}$ \\
\hline $\begin{array}{l}\text { Balaenoptera acutoro- } \\
\text { strata scammoni }\end{array}$ & South Korea & NA & $303^{a}$ & $1996-2008$ & $20,22,24,498-501$ \\
\hline $\begin{array}{r}\text { Balaenoptera } \\
\text { bonaerensis }\end{array}$ & All (Antarctic) & NA & Unk. & & \\
\hline \multirow{6}{*}{$\begin{array}{l}\text { Megaptera } \\
\text { novaeangliae }\end{array}$} & South America & $<1$ & $<5^{\mathrm{b}}$ & 1995-2005 & $8,14,166,197,421$ \\
\hline & Northwest Atlantic & $5-20$ & $<3^{b}$ & 1990-2009 & $\begin{array}{l}72,275,277,313-315,328-333 \\
545,547,548,551-553\end{array}$ \\
\hline & Arabian Sea & NA & $<1$ & 1990-2009 & 63,354 \\
\hline & Zanzibar & NA & $4^{\mathrm{a}}$ & 2002-2004 & $17,292,439$ \\
\hline & South Africa & NA & $<3^{\mathrm{b}}$ & $1994-2008$ & 352,398 \\
\hline & Australia & NA & $<3^{\mathrm{b}}$ & $1992-2006$ & $\begin{array}{l}27,103,215,216,230,232,245 \\
256,429\end{array}$ \\
\hline \multicolumn{6}{|l|}{ Eschrichtiidae } \\
\hline Eschrichtius robustus & Northeast Pacific & Low 10s & $<10^{\mathrm{b}}$ & 1990-2009 & $\begin{array}{l}13,33,35,59,201,255,279 \\
518,563\end{array}$ \\
\hline \multicolumn{6}{|l|}{ Neobalaenidae } \\
\hline Caperea marginata & $\begin{array}{l}\text { All (circumpolar in } \\
\text { Southern Hemisphere) }\end{array}$ & $<1$ & $4^{\mathrm{a}}$ & $2002-2003$ & 103,147 \\
\hline
\end{tabular}

Table S2). The numbers recorded for most species are small in relation to population sizes and geographic ranges. A major difference between the large mysticetes and the odontocetes in general is the degree to which mysticetes are prone to lethal entanglement in ropes and lines as well as net mesh. An important further consideration is that some mysticete populations (e.g. North Atlantic and North Pacific right whales, southern right whales in the southeastern Pacific off Chile and Peru, humpback whales in the northern Arabian Sea) are small and endangered, and, for them, even small numbers of entanglements are potentially significant (see, for example, Minton et al. 2011).

Humpback whales, right whales, and minke whales become entangled in gillnets relatively often, at least in some areas, even though this is not always obvious from bycatch mortality statistics, per se. In a study of 
61 humpback and right whale entanglements in fishing gear off eastern North America between 1993 and 2002, the gear type was determined in 36 cases (22 humpback, 14 right) (Johnson et al. 2005). Of those, 11 humpback and 2 right whale entanglements involved gillnets, i.e. 50 and $14 \%$, respectively. The high rates of scarring on living whales confirm that entanglement occurs much more frequently than implied by the statistics on known bycatch mortality (Knowlton et al. 2008, Robbins 2009). Assignment of scars to particular gear types is often not possible, and therefore it is difficult to determine the relative frequency of entanglement in gillnets as opposed to entanglement in other gears. Gillnet entanglement of humpback whales has long been considered a serious problem in Ecuador, which is said to have the largest artisanal fishing fleet in the southeastern Pacific (Félix et al. 2011). Similar problems of humpback whale entanglement exist in other regions, e.g. Colombia (Flórez-González \& Capella 2010) and the western Indian Ocean off Zanzibar (Amir et al. 2012).

In South Africa, entanglement of southern right whales in gillnets appears to be infrequent (Best et al. 2001), whereas entanglements of both right whales and humpback whales in large-mesh shark control nets off Kwazulu-Natal occur frequently, at different seasons (Meÿer et al. 2011). An active disentanglement program has existed since 1990, and this has reportedly reduced substantially the mortality of bycaught animals (Meÿer et al. 2011).

Minke whales are probably especially vulnerable to gillnet entanglement for several reasons, including their near-shore and shelf occurrence, their proclivity for preying on fish species that are also targeted by net fisheries, and their small size and consequently greater difficulty (compared to the larger mysticetes) of extricating themselves once caught. A thorough review of minke whale records for southern parts of the eastern North Atlantic found evidence of net entanglement in the Azores, Canary Islands, and Senegal, and the authors of the study (Van Waerebeek et al. 1999) concluded that the general problem of incidental mortality of minke whales has 'received little attention and both its true extent and the impact on populations remain unassessed.' It seems likely that gillnet entanglement of minke whales, as well as the mid-sized rorquals with coastal distributions such as Bryde's whales Balaenoptera edeni/brydei and Omura's whales $B$. omurai, occurs much more often than suggested by the available statistics. Also, in southern California in the 1980s, when coastal gillnetting was common (it has since been prohibited in California), gray whale Eschrichtius robustus entan- glement was relatively frequent, with 61 events (20 dead whales, 41 alive) documented between 1981 and 1989 (Heyning \& Lewis 1990).

The only country with good data for relatively large and regular bycatches of mysticetes in recent years is South Korea, where the total reported gillnet catch of minke whales was 303 between 1996 and 2008. The true catch was probably considerably higher, given the results of sampling and analyses of meat sold in Korean markets in the late 1990s and early 2000s (Baker et al. 2007). Of 214 investigated entanglements of minke whales in the Sea of Japan (East Sea) between 2004 and 2007, about 65, or $30 \%$, of the whales were judged to have been caught in float lines from gillnets (Song et al. 2010). The concept of 'bycatch' in South Korea is confounded by the high commercial value of whale meat and the possibility that nets are sometimes deployed with intent to capture whales (MacMillan \& Han 2011). Importantly, as well, the minke whales taken in Korean waters are part of an unusual autumn-breeding population, the Sea of Japan-Yellow Sea-East China Sea stock, commonly known as J-stock, which has been of concern for many years in view of historical removals by whaling, ongoing 'research whaling' by Japan, and the relatively large ongoing bycatches in Korea, Japan, and possibly China (Reilly et al. 2008).

Bycatches of mysticetes in Chinese waters likely occur more often than suggested by the available literature. In 1990 there were an estimated 10000 driftnet vessels and 7000 set gillnet vessels operating in Chinese coastal waters (IWC 1994, p. 19). However, we were able to locate only 2 records of gillnet bycatch in China since 1990 - a minke whale (misreported as a gray whale calf in Chinese media) in 2008 and an adult gray whale in 2011 (Zhu 2012).

\section{Phocid seals}

Fifteen of the 18 extant species of phocid seals were taken as bycatch between 1990 and 2011, and, of those, 14 were captured in gillnets (Table 4, Table S3).

The ringed seal subspecies Pusa hispida saimensis, endemic to Lake Saimaa in Finland, may number only a few hundred, and entanglement in nets, including gillnets, is considered the most serious threat to the population (Sipilä \& Hyvärinen 1998, Sipilä 2003). The same may be true of the more numerous Lake Ladoga subspecies $P$. h. ladogensis. It was estimated in the early 1990 s that 200 to 400 Ladoga seals from a total population of at least 5000 
Table 4. As in Table 2 but for illustrative pinniped bycatch. For all further details see Table 2 legend

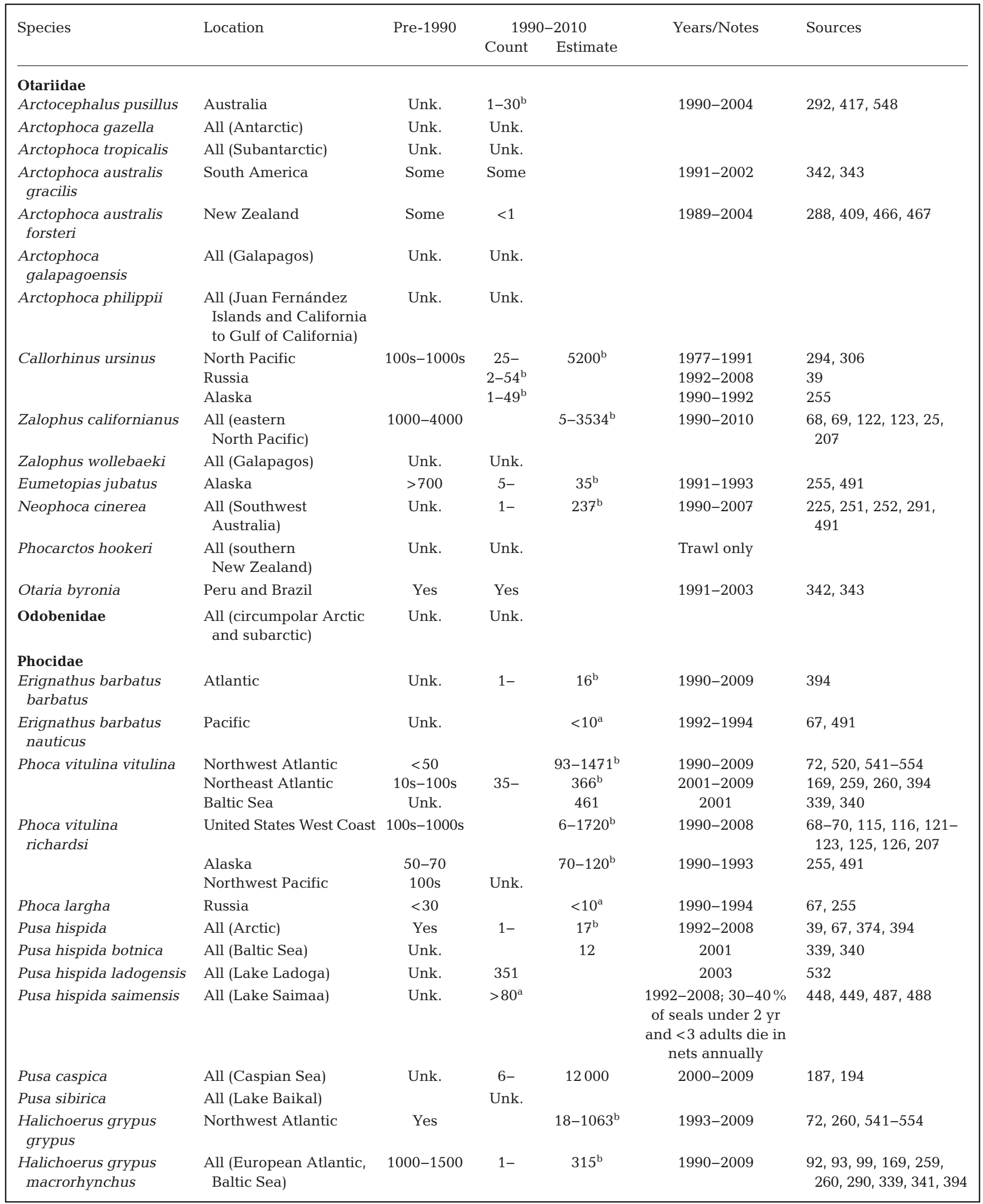


Table 4 (continued)

\begin{tabular}{|c|c|c|c|c|c|c|}
\hline \multirow[t]{2}{*}{ Species } & \multirow[t]{2}{*}{ Location } & \multirow[t]{2}{*}{ Pre-1990 } & \multicolumn{2}{|c|}{$1990-2010$} & \multirow[t]{2}{*}{ Years/Notes } & \multirow[t]{2}{*}{ Sources } \\
\hline & & & Count & Estimate & & \\
\hline Histriophoca fasciata & Russia & $10 \mathrm{~s}-100 \mathrm{~s}$ & $32-310^{\mathrm{b}}$ & & $1992-2008$ & 39 \\
\hline $\begin{array}{l}\text { Pagophilus } \\
\text { groenlandicus }\end{array}$ & $\begin{array}{l}\text { All (North Atlantic } \\
\text { and Arctic) }\end{array}$ & $\begin{array}{l}1000 s- \\
100000\end{array}$ & & $100 \mathrm{~s}-46394^{\mathrm{b}}$ & 1990-2009 & $\begin{array}{l}259,260,394,489 \\
541-554\end{array}$ \\
\hline Cystophora cristata & $\begin{array}{l}\text { All (North Atlantic } \\
\text { and Arctic) }\end{array}$ & Yes & $1-$ & $82^{\mathrm{b}}$ & 1995-2008 & $394,542,548,549$ \\
\hline Monachus schauinslandi & All (Hawaii) & Some & Unk. & $<1^{\mathrm{b}}$ & $1994-2007$ & 69,125 \\
\hline Mirounga leonina & $\begin{array}{l}\text { All (circumpolar in } \\
\text { Southern Hemisphere) }\end{array}$ & Unk. & 1 & & 1998 & 288 \\
\hline Mirounga angustirostris & $\begin{array}{l}\text { All (Central and Eastern } \\
\text { North Pacific) }\end{array}$ & $<10$ & $5-$ & $314^{\mathrm{b}}$ & 1990-2003 & $68,69,122,126,207$ \\
\hline Leptonychotes weddellii & $\begin{array}{l}\text { All (circumpolar in } \\
\text { Southern Hemisphere) }\end{array}$ & Unk. & Unk. & & & \\
\hline Lobodon carcinophaga & $\begin{array}{l}\text { All (circumpolar in } \\
\text { Southern Hemisphere) }\end{array}$ & Unk. & Unk. & & & \\
\hline Hydrurga leptonyx & $\begin{array}{l}\text { All (circumpolar in } \\
\text { Southern Hemisphere) }\end{array}$ & Unk. & $2^{\mathrm{a}}$ & & Unk.-1998 & 288 \\
\hline
\end{tabular}

died annually in fishing gear, but 'Since 1992 it has become increasingly difficult to estimate the bycatch of (Ladoga ringed) seals because small scale netting has become more common' (Sipilä \& Hyvärinen 1998, p. 93).

Although the endangered Caspian seal is subject to numerous types of threats, living as it does in an enclosed water body subject to intensive human use, bycatch in illegal sturgeon gillnets has been identified as a major threat, with perhaps 12000 or more taken in these nets each year (Dmitrieva et al. 2011). This level of gillnet bycatch would represent close to $10 \%$ of the estimated current total population of the species (approximately 100000; Harkonen et al. 2012).

Gillnet bycatch is one of several serious threats to the Critically Endangered Mediterranean monk seal, of which fewer than 250 mature individuals may remain (Aguilar \& Lowry 2008), even though there are few confirmed records and no credible estimates of bycatch derived from data. Karamanlidis et al. (2008) reviewed available evidence, particularly for Greek waters between 1991 and 2007, and concluded that gillnet mortality was a major threat to the species. Subadults appeared particularly prone to entanglement; most of the documented mortality of adults was caused by deliberate killing. Those authors also concluded that a resurgence of gillnetting off Cap Blanc, NW Africa, was a growing con- cern. In the Foça Pilot Monk Seal Conservation Area along the Aegean Sea coast of Turkey, seal interactions with fisheries were observed between 1994 and 2002. Four entanglements were documented in trammel nets, and 2 in gillnets, all non-fatal, although on 1 occasion the animal and the gear had to be taken ashore in order to cut the animal free (Güçlüsoy 2008).

Thousands to 10000 s of harp seals are caught in gillnets in Canada each year, but the population is in the millions, and the removal rate is considered sustainable (DFO 2011). Many 100s, possibly 1000s, of gray seals and harbor seals are taken annually as bycatch in gillnet fisheries in the North Atlantic, but in much of the range (including the North Pacific in the case of harbor seals) no effort is made to document and report levels (e.g. NAMMCO 2007). There are suggestions that in some areas (e.g. Norway) the bycatch of harbor seals has been high enough to reduce populations, particularly when considered in combination with deliberate killing (see papers in Desportes et al. 2010).

\section{Otariid seals and sea lions}

Of the 14 species of otariid seals and sea lions (including 1 extinct species), 8 are known to have been bycaught, 7 of them in gillnets, between 1990 
and 2011 (Table 4, Table S3). Since the time when Woodley \& Lavigne (1991) reported bycatch in passive gear to be a major cause of population decline for northern fur seals, the high-seas driftnet fisheries for salmon and flying squid, which accounted for the deaths of 1000 s of fur seals annually, have largely stopped. The estimated kill in the driftnet fisheries in 1991 was 5200 (95\% CI = 4500 to 6000) (Larntz \& Garrott 1993). Although some illegal driftnetting may still occur, it is generally assumed that recent levels of bycatch of this species have been low (COSEWIC 2010, Allen \& Angliss 2011).

Steller sea lions and New Zealand sea lions, the only other otariids cited by Woodley \& Lavigne (1991) as being at significant risk from bycatch, were and continue to be affected at least as much by mortality in trawl nets as in gillnets (Allen \& Angliss 2011, Robertson \& Chilvers 2011). It appears from the literature reviewed that, although these animals certainly are vulnerable to bycatch in gillnets, the population-level threat from such bycatch is small in comparison to that from trawl bycatch.

Australian sea lions Neophoca cinerea are endangered primarily because of bycatch in demersal gillnet and trap fisheries (Goldsworthy \& Page 2007, Goldsworthy \& Gales 2008). Gillnet fishing for sharks overlaps significantly with the range of sea lions, and bycatch levels continue to be high enough to threaten the species despite relatively intensive efforts to manage the fishery for bycatch reduction (Hamer et al. 2011, 2013). Many, perhaps most, of the sea lions taken in gillnets become entangled while engaged in depredation on small sharks caught in the nets (Hamer et al. 2011). There are 2 important considerations when evaluating the accuracy of bycatch statistics in this fishery. First, some animals manage to escape with severe and sometimes lifethreatening injuries (similar to the situation mentioned earlier for baleen whales); their deaths are unlikely to be included in bycatch statistics (Hamer et al. 2011, 2013). Second, an unknown proportion of the sea lions that have died in the nets drop out before being detected, even by a vigilant onboard observer (Hamer et al. 2011, 2013).

In terms of the sheer scale of gillnet bycatch, California sea lions Zalophus californianus deserve mention. Woodley \& Lavigne (1991) cited an estimate of around 2250 being killed in gillnets annually in California alone 'in a typical year,' and we have no evidence to suggest a strong decline (or increase) in such mortality since then; certainly at least 100s still die annually in gill and other entangling nets (Carretta et al. 2011).
Pinniped depredation is a major problem at many aquaculture facilities in Europe, Chile, the United States, Australia, and South Africa (Kemper et al. 2003). Anti-predator nets are commonly used as a deterrent. Although seals and sea lions are bycaught only infrequently (i.e. die from becoming entangled in an anti-predator net or from becoming trapped between it and the main cage or pen), many pinnipeds are directly harassed and killed as pests by aquaculture operators (Kemper et al. 2003).

\section{Odobenidae}

We found no published evidence of walrus bycatch between 1990 and 2011.

\section{Sirenians}

All 4 sirenian species were taken as bycatch in gillnets between 1990 and 2011 (Table 5, Table S4).

Bycatch data for sirenians, particularly manatees, are extremely scarce. With very few exceptions, all that is available comes from anecdotal reports of observed catches, observations of animals (living or dead) with gear on the body or wounds clearly attributable to gillnet gear, or general statements in the literature that bycatch occurs. In Australia the dugong bycatch in shark control nets was monitored between 1962 and 1992 when it averaged about $27 \mathrm{yr}^{-1}$; with mitigation measures in place after 1992 the rate declined to about $2 \mathrm{yr}^{-1}$ (Marsh et al. 2002). An intensive interview study by Jaaman et al. (2009) is a singular example of an attempt to estimate the total sirenian bycatch over a large area, in this case that of dugongs in artisanal gillnets in East Malaysia. Those authors estimated that 479 (95\% CI = 434 to 528) dugongs were bycaught in Sabah per year from 1997 to 2004, and they considered this estimate to be negatively biased. However, as is often true of sirenians, the value of the animals to local people for nutritional and cultural uses created ambiguity in how bycatch was defined and recorded. Jaaman et al.'s fisherman informants described the dugong as 'the main marine mammal species hunted in Sabah waters.' It is frequently impossible to tease apart true bycatch from the often deliberate netting in what are not really 'gill'-nets per se, but large-mesh entangling nets set to catch fairly large edible creatures including sirenians (e.g. Reeves et al. 1988, 1996, Dodman et al. 2008).

Even though very little quantitative information is available on gillnet bycatch of sirenians, we suspect it is a serious threat to numerous populations. 
Table 5. As in Table 2 but for illustrative sirenian and mustelid bycatch. For all further details see Table 2 legend

\begin{tabular}{|c|c|c|c|c|c|}
\hline \multirow[t]{2}{*}{ Species } & \multirow[t]{2}{*}{ Location } & \multicolumn{2}{|c|}{ 1990-2010 } & \multirow[t]{2}{*}{ Years/Notes } & \multirow[t]{2}{*}{ Sources } \\
\hline & & Count & Estimate & & \\
\hline \multicolumn{6}{|l|}{ Trichechidae } \\
\hline \multirow[t]{3}{*}{ Trichechus manatus } & Florida & Some & & $1990-2007$ & 213 \\
\hline & Caribbean Sea & Some & & Early 1990s & $54,72,113,319,353,361$ \\
\hline & Brazil & $13^{\mathrm{a}}$ & & 1987-2002 & 173,410 \\
\hline Trichechus senegalensis & Guinea-Bissau & $185^{\mathrm{a}}$ & & 1990-1998 & 484 \\
\hline \multirow[t]{3}{*}{ Trichechus inunguis } & Brazil & $6^{\mathrm{a}}$ & & 1990-2002 & 413 \\
\hline & Colombia & $6^{\mathrm{a}}$ & & $2000-2005$ & 349 \\
\hline & Orinoco River & $39^{a}$ & & 1980-2004 & 127,438 \\
\hline \multicolumn{6}{|l|}{ Dugongidae } \\
\hline \multirow[t]{6}{*}{ Dugong dugon } & United Arab Emirates & & $15^{\mathrm{b}}$ & Early 1990s & 61,62 \\
\hline & East Africa & $1-24^{\mathrm{b}}$ & & 1990-2004 & $\begin{array}{r}18,145,155,156,170,241,292 \\
293,351,365,428,432,539,557\end{array}$ \\
\hline & Northern Indian Ocean & $1-20^{\mathrm{b}}$ & & 1990-2006 & $265-267$ \\
\hline & Thailand & $40^{\mathrm{a}}$ & & 1990-1995 & 6 \\
\hline & Malaysia & $4-$ & $493^{b}$ & 1994-2004 & 273,351 \\
\hline & Australia & $1-36^{\mathrm{b}}$ & & 1990-2010 & $\begin{array}{l}27,55,91,229,231,234,235 \\
245,335,351\end{array}$ \\
\hline \multicolumn{6}{|l|}{ Mustelidae } \\
\hline Enhydra lutris lutris & Northwest Pacific & $3^{\mathrm{a}}$ & & 1996-2001 & $252,270,301$ \\
\hline Enhydra lutris kenyoni & Northeast Pacific & $1-12^{\mathrm{b}}$ & & 1990-2007 & 37 \\
\hline Enhydra lutris nereis & Central California & $1-$ & $64^{\mathrm{b}}$ & 1990-2005 & 37,206 \\
\hline Lontra felina & Peru & $1-2^{\mathrm{b}}$ & & 1991-2008 & 343 \\
\hline
\end{tabular}

\section{Otters}

Both sea otters and marine otters were taken as bycatch in gillnets between 1990 and 2011 (Table 5, Table S4). A 1991 ban on gill and trammel net fishing in California waters shallower than $30 \mathrm{~m}$ substantially reduced the sea otter bycatch, from around 80 to $100 \mathrm{yr}^{-1}$ in the $1980 \mathrm{~s}$ to $<50 \mathrm{yr}^{-1}$ in the 1990 s (Forney et al. 2001). By the early 2000s, the bycatch of sea otters in California was thought to be near zero (Forney et al. 2001). In Alaska, sea otters are at risk of entangling and drowning in salmon gillnets in a number of areas. For example, it was estimated that $<10$ died per year in the early 2000s in the salmon set net fishery at Kodiak Island (Allen \& Angliss 2011).

\section{DISCUSSION AND CONCLUSIONS}

\section{Methodological issues}

There has been a strong and persistent theme in the marine mammal bycatch literature over the past 20-plus years emphasizing the paramount importance of onboard observer programs to obtain credible estimates of bycatch levels and rates. For exam- ple, 'It is widely accepted that accurate estimation of bycatch rates in any fishery requires an independent observer scheme' (Read et al. 2006, p. 167). Artisanal fisheries present special challenges in this regard, in part because vessels often do not have space and other accommodation to carry more than the crew. In a number of areas, however, researchers have managed to place observers on at least a small sample of artisanal fishing boats to obtain index values for bycatch rates (Mangel et al. 2010, Bjørge et al. 2011, O. A. Amir \& P. Berggren unpubl.). Such values, when used in combination with data on fishing effort, observations of landings, and information from interviews with fishermen, have greatly enhanced the accuracy of bycatch estimates for those fisheries.

As long as onboard observer programs (or alternatives such as having patrol vessels at sea to monitor net hauls or the use of video monitoring) remain exceptional rather than typical (which seems likely), the available estimates or indices of marine mammal bycatch for a great many of the world's artisanal fisheries will continue to come from other approaches, ranging from opportunistic observations at ports of landing, to studies of scarring and injuries on live or dead animals encountered at sea, 
to studies of strandings, and to formal fisherman interview programs.

It is often impossible to identify, quantify, and account for bias in data obtained from such approaches. For example, in a broad-scale interview study by Moore et al. (2010), the authors acknowledged that marine mammal and sea turtle bycatch was, on the one hand, likely under-reported in countries where it was illegal, but, on the other hand, possibly overreported (in other countries?) 'to impress interviewers, comply with perceived interviewer attitudes, or if they (the interviewed fishermen) perceive(d) opportunities to attract outside investment in their communities.'

Efforts to define, quantify, assess, and mitigate the bycatch threat are confounded by the fact that some of the mortality and serious injury in fishing gear is due to 'debris,' which includes derelict or discarded gear ('ghost fishing') (not to mention packing material and trash from fishing vessels and aquaculture facilities) (Laist 1997, Laist et al. 1999, Kemper et al. 2003, Page et al. 2004, Raum-Suryan et al. 2009). As observed by Laist (1996, p. 33):

Although ghost fishing and entanglement are not usually considered part of the bycatch issue, they catch many of the same species taken as bycatch. The only real difference is that one involves derelict fishing gear and the other involves active gear. In this sense, ghost fishing and entanglement are related parts of the same basic problem - namely, preventing extraneous mortality of marine life in fishing gear.

However, bycatch estimates generated from fishery observer data, logbook or recall (questionnaire) programs, market surveys, etc. are unlikely to reflect the debris component, whereas those generated from strandings might do so, at least to an extent. Moreover, in studies of 'entanglement rates' based on scars, wounds, or gear on the body, such as analyses of photographs of living whales at sea (Robbins 2009, Knowlton et al. in press), observations of pinnipeds on shore (Fowler 1987, Page et al. 2004, RaumSuryan et al. 2009), and stranded carcasses (Pinedo \& Polachek 1999), the distinction between in-use gear and discarded/lost gear is necessarily obscured.

A recent study in the Black Sea found a correlation between the quantity of marine debris (defined in terms of both number of items and estimated weight per kilometer of netting) and number of harbor porpoises brought to the surface in bottom-set gillnets targeting turbot Psetta maxima maeotica (Birkun \& Krivokhizhin 2008). The authors proposed as a working hypothesis to explain this finding that as plastic debris accumulates on the sea bottom, it functions as an artificial substrate for benthic organ- isms, attracting concentrations of fish and crustaceans that in turn attract higher order predators including cetaceans. The ultimate effect of such a process on marine mammal populations would be difficult to gauge, as, on the one hand, it could enhance foraging success, but, on the other, bring greater risk of entanglement.

\section{Patterns in availability of marine mammal bycatch data}

Much of what is known about marine mammal bycatch reflects the distribution and nature of research effort, including observer programs. That is, we learn about the occurrence of bycatch largely as a result of someone being present at the site to observe and report it. For information on scale and other details, we often must depend on someone (e.g. from a non-governmental organization or government agency) being present in the area with the necessary interest (or in the case of government agencies, the mandate) to pursue questions beyond the simple fact of occurrence.

It is difficult to see how this type of bias can be overcome other than by continuing to promote and support (financially and technically) projects or programs that get more people into the field, especially in areas of known spatial and temporal overlap between marine mammals and fisheries. The alternative is to develop or refine methods of projecting bycatch estimates for unsampled areas by applying bycatch rate estimates obtained in 'equivalent' areas to data on marine mammal occurrence and fishery effort in those unsampled areas, as pioneered by Read et al. (2006).

The bycatch literature suggests a frequent pattern of problem discovery, followed by intense investigation and building of awareness, followed by a dropping-off of interest or focus as either (1) funding stops, (2) the researcher's interest or ability to pursue the topic recedes (e.g. after his/her academic degree work has been completed), (3) other priorities emerge and overtake this one, or (4) authorities manage to rationalize or conceal the problem. For example, local and foreign scientists working in Sri Lanka in the early 1980s discovered from observations at fish landing sites and markets that large numbers of cetaceans were being caught 'incidentally' in local fisheries (Leatherwood \& Reeves 1989). Estimates of the scale of this bycatch varied (Leatherwood 1994), but the 1990 IWC workshop concluded that $>40000$ cetaceans may have been killed annually in Sri 
Lankan artisanal gillnet fisheries at the time (IWC 1994, p. 15). Foreign scientists stopped visiting Sri Lanka after the 1980s, but a study in 1991/1992 by Sri Lankan scientists confirmed that at least 1000s of dolphins and small whales were still being landed and sold for human consumption each year (Dayaratne \& Joseph 1993). Since the early 1990s, no new information has become available. When asked to help us identify sources of new information, one of the researchers involved in bycatch investigations in the 1980s replied that there had been no formal or consistent monitoring or targeted bycatch studies in Sri Lanka since the early 1990s, and that the few marine mammal researchers in the country were instead using the scarce available resources to study living animals at sea (Anouk Ilangakoon, pers. comm., 15 July 2011). She confirmed, however, from casual observations that the bycatch situation in Sri Lanka had not changed over the last 2 decades.

In fact, available information on the scale of gillnet fishing in Sri Lanka (possibly as many as 46000 vessels, annual gillnet catches of about $153000 \mathrm{t}$ from 2006 to 2010; MRAG 2012) supports the inference of a continuing high level of cetacean bycatch there. Moreover, the estimated annual fish catch by the tuna gillnetting fleet from Iran is even higher than Sri Lanka's, and the annual catch levels by tuna gill-netting fleets from Indonesia, India, Pakistan, Oman, and Yemen are all $>20000$ t (MRAG 2012). The bycatch of cetaceans in all of these Indian Ocean countries is unmonitored and likely high enough to merit conservation concern. It is clear that a major data gap exists in the northern Indian Ocean and that improved marine mammal bycatch reporting from gillnet fisheries in that region should be a global priority. The same can be said of the Pacific coasts of Mexico, Central America, and South America, as well as the east (Indian Ocean) and west (Atlantic) coasts of Africa.

The situation in Sri Lanka is complicated by the fact that cetaceans are widely valued there as food (Ilangakoon et al. 2000), which is also the case in Peru (Van Waerebeek \& Reyes 1994a, Van Waerebeek et al. 1997), the Philippines (Dolar et al. 1994), Ghana (Van Waerebeek et al. 2009), and other areas. In Ecuador (and elsewhere), 'fishermen know that dolphin meat is excellent bait on their longlines and they are willing to pay a lot of money for bycatch' (Félix \& Samaniego 1994). Such usage obscures the distinction between accidental and deliberate catches. Also, it can mean there is less incentive for consistent and reliable reporting when, as is often the case, the deliberate capture of cetaceans is ille- gal. The use of cetaceans as food appears to be increasing in some parts of the world (Robards \& Reeves 2011), reminiscent of the 'bush meat' trade in Africa, and thus the concept of 'marine bush meat' has developed (Clapham \& Van Waerebeek 2007).

Another pattern, much less frequent, is where, following initial problem discovery and definition, efforts to document bycatch continue for decades as successive researchers piece together funding from various sources (often both non-governmental and governmental) and manage to keep the bycatch issue from being suppressed or ignored. In Peru, for example, a team sponsored by the United Nations Environment Program provided initial quantitative documentation of the nature and scale of the cetacean bycatch problem in 1985 and 1986 (Read et al. 1988). Follow-up efforts by dedicated individuals and small groups of researchers have provided some level of monitoring, including studies explicitly intended to assess the effectiveness of legal measures taken by the Peruvian government to reduce cetacean mortality in fisheries in response to the revelations in the 1980s (Van Waerebeek \& Reyes 1994b, Van Waerebeek et al. 2002, Mangel et al. 2010).

There are some examples, unfortunately rare, of a different pattern, in which problem discovery is followed by intense investigation and awareness building, followed by serious and sustained efforts to address and solve the problem, accompanied by ongoing monitoring to assess effectiveness. This pattern can be said to apply to parts of western Europe (e.g. through working groups of ICES, the International Council for Exploration of the Sea, and the 2 cetacean-oriented agreements under CMS, the Convention on Migratory Species), Australia (Goldsworthy \& Page 2007, Goldsworthy et al. 2007, Hamer et al. 2011, 2013), and New Zealand (Dawson \& Slooten 1993, Slooten \& Dawson 2010, Gormley et al. 2012), as well as the United States (Carretta et al. 2008, Carretta \& Barlow 2011, Orphanides \& Palka 2013, this Theme Section, Read 2013, this Theme Section). However, even in those regions, it is a challenge to maintain monitoring and mitigation efforts in view of the costs and the need to reinforce the idea that gillnet bycatch is a credible and ongoing threat to many marine mammal populations.

In the absence of legal and governance regimes that require and enable regular monitoring, bycatch documentation in much of the world is likely to remain patchy, far from complete, and largely idiosyncratic. Given the prohibitively high cost of onboard observer programs, or even of programs 
such as those in Peru and Norway where index values have been obtained for fleet-wide extrapolations, there is need for much wider application of a relatively low-cost, rapid approach to bycatch assessment such as that taken by Moore et al. (2010).

\section{Bycatch as a continuing (increasing?) threat to marine mammal species and populations}

Assessing the seriousness of gillnet bycatch as a threat to marine mammal diversity and abundance is a complex task. It depends, at least in part, on how diversity and abundance are defined. A number of recent studies have explored ways of determining biodiversity conservation priorities on the basis of phylogenetic distinctiveness (e.g. Isaac et al. 2007, Pyenson 2009, May-Collado \& Agnarsson 2011). These help to underscore not only the relative evolutionary significance of the extinction of the baiji, but also the urgency of conserving certain other species with long, diverse lineages and few or no extant sister taxa, such as the obligate freshwater dolphins of the South Asian subcontinent (Platanista) and South America (Inia), the franciscana, the Mediterranean monk seal, and the finless porpoises (Neophocaena), all of which, as shown in the present study, are threatened in all or parts of their range by gillnet bycatch.

Another factor that complicates efforts to assess the seriousness of gillnet bycatch as a threat to species and populations is the array of biases in documentation and reporting. As mentioned earlier, many species, but particularly freshwater, estuarine, and coastal marine species that co-occur with large artisanal gillnetting fleets in developing countries, are clearly vulnerable to unsustainable bycatch, yet in most instances quantitative data on the scale of mortality, as well as on the marine mammal populations, are fragmentary and far from complete. The paucity of data is due not only to the lack of institutional and legal commitments, but also to problems of personal security for researchers, the non-availability of infrastructure for mounting observational studies, and the chronic inadequacy of funding. Such obstacles create or add to the risk that some, probably many, serious situations will continue to be under-appreciated or missed altogether.

Finally, knowing the level or rate of bycatch alone is insufficient for assessing the scale and immediacy of the threat. Proper assessment requires, at a minimum, an understanding of population structure and a credible estimate of current population size. In addi- tion, it is important to know something about the population's range, individual movement patterns, source-sink dynamics, and relative vulnerability of different sex or age classes. Such data requirements are rarely met, even in highly developed countries with strong legal and institutional foundations (Taylor et al. 2007).

A trend that is often overlooked is the proliferation of aquaculture operations for finfish (especially salmon), mollusks, seaweed, and other species. Since 1990, annual production of salmonid farms has increased from 299000 to 1900000 t (FAO 2012), and accompanying this expansion has been an increase in conflicts with marine mammals, especially pinnipeds. In some aquaculture operations, bycatch of marine mammals in anti-predator nets occurs at least occasionally, although direct killing and exclusion from preferred habitat may represent more serious problems for the marine mammal populations (Kemper et al. 2003).

Perhaps the most important finding of this study is that, some 20-plus years after the landmark IWC workshop (Perrin et al. 1994), the threat of bycatch in passive fishing gear is far from resolved and is likely growing rather than receding. The remarkable shortage of rigorous, comprehensive bycatch accounting (e.g. long time series of annual estimates by species, stock, area, or fishery) was an unexpected and disappointing finding. There is a danger that other ongoing or looming threats, including bycatch in other types of fishing gear (e.g. trawls, purse seines, longlines), as well as habitat deterioration, vessel strikes, novel disease outbreaks, ingestion of plastic debris, overfishing of prey species, and the intractable effects of global climate change, will be allowed to overshadow the nagging, persistent threat of marine mammal bycatch in passive fishing gear, particularly for already threatened coastal species and small populations.

Acknowledgements. This work was undertaken to provide background for a workshop on bycatch mitigation in October 2012, organized by the Consortium for Wildlife Bycatch Reduction under NOAA Grant No. NA09NMF4520413. Additional support was received from the Lenfest Ocean Program through a grant to the New England Aquarium. Also, it should go without saying that we benefited from the generous advice and input from the many colleagues who responded to our requests for references, clarifications, corrections, and, in some cases, data. We specifically thank Bob Brownell, Per Berggren, and an anonymous reviewer for their helpful comments on the manuscript. We are also indebted to Bill Perrin for many things, including help with references for this paper and his decades-long leadership in bringing global attention to the bycatch issue. 


\section{LITERATURE CITED}

Aguilar A, Gaspari S (2012) Stenella coeruleoalba (Mediterranean subpopulation). In: IUCN 2012. IUCN Red List of Threatened Species, Version 2012.1. IUCN, Gland. Available at www.iucnredlist.org (accessed 14 October 2012)

Aguilar A, Lowry L (2008) Monachus monachus. In: IUCN 2011. IUCN Red List of Threatened Species, Version 2011.2 IUCN, Gland. Available at www.iucnredlist.org (accessed 24 April 2012)

Allen BM, Angliss RP (2011) Alaska marine mammal stock assessments, 2010. NOAA Tech Memo NMFS-AFSC 223:1-292

Amir OA, Berggren P, Jiddawi NS (2012) Recent records of marine mammals in Tanzanian waters. J Cetacean Res Manag 12:249-253

Andersen MS, Forney KA, Cole TVN, Eagle T and others (2008) Differentiating serious and non-serious injury of marine mammals: report of the Serious Injury Technical Workshop. US Dept Commerce, NOAA Tech Memo NMFS-OPR 39:1-94

Baker CS, Cooke JG, Lavery S, Dalebout ML and others (2007) Estimating the number of whales entering trade using DNA profiling and capture-recapture analysis of market products. Mol Ecol 16:2617-2626

Baldwin RM, Collins M, Van Waerebeek K, Minton G (2004) The Indo-Pacific humpback dolphin of the Arabian region: a status review. Aquat Mamm 30:111-124

Benjamins S, Lawson J, Stenson G (2007) Recent harbor porpoise bycatch in gillnet fisheries in Newfoundland and Labrador, Canada. J Cetacean Res Manag 9:189-199

Best PB (2007) Whales and dolphins of the southern African subregion. Cambridge University Press, Cambridge

Best PB, Peddemors VM, Cockcroft VG, Rice N (2001) Mortalities of right whales and related anthropogenic factors in South African waters, 1963-1998. J Cetacean Res Manag 2(Spec Issue):161-169

Birkun AA Jr, Frantzis A (2008) Phocoena phocoena ssp. relicta. In: IUCN 2011. IUCN Red List of Threatened Species, Version 2011.2. IUCN, Gland. Available at www.iucnredlist.org (accessed 7 April 2012)

Birkun AA Jr, Krivokhizhin S (2008) Involvement of Black Sea artisanal fisheries in anti-bycatch and anti-marine litter activities. Report to the Permanent Secretariat of the Commission on the Protection of the Black Sea against Pollution and Permanent Secretariat of the Agreement on the Conservation of Cetaceans of the Black Sea, Mediterranean Sea and contiguous Atlantic Area. Black Sea Council Marine Mammals, Simferopol

Bjørge A, Godøy H, Skern-Mauritzen M (2011) Estimated bycatch of harbour porpoise Phocoena phocoena in two coastal gillnet fisheries in Norway. Document SC/63/ SM18. Available from Secretariat, International Whaling Commission, Cambridge

Bravo C, Pérez MJ, Barría P, Bustos G and others (2010) Implementación de acciones para la conservación del delfín chileno, Cephalorhynchus eutropia, en la zona de Constitución, region del Maule, Chile. In: Félix F (ed) Esfuerzos para mitigar el impacto de actividades pesqueras en cetáceos en los países del Pacífico Sudeste. Comisión Permanente del Pacífico Sur, Guayaquil, p 3-10

Buckland S, Cattanach K, Hobbs R (1993) Abundance estimates of Pacific white-sided dolphin, northern right whale dolphin, Dall's porpoise and northern fur seal in the North Pacific, 1987-1990. Bulletin INPFC 53:387-407

Carretta JV, Barlow J (2011) Long-term effectiveness, failure rates, and "dinner bell' properties of acoustic pingers in a gillnet fishery. Mar Technol Soc J 45(5):7-19

Carretta JV, Barlow J, Enriquez L (2008) Acoustic pingers eliminate beaked whale bycatch in a gillnet fishery. Mar Mamm Sci 24:956-961

Carretta JV, Forney KA, Oleson E, Martien K and others (2011) U.S. Pacific marine mammal stock assessments: 2010. NOAA Tech Memo NMFS-SWFSC 476:1-352

Clapham P, Van Waerebeek K (2007) Bushmeat and bycatch: the sum of the parts. Mol Ecol 16:2607-2609

Cockcroft VG (1990) Dolphin catches in the Natal shark nets, 1980 to 1988. S Afr J Wildl Res 20:44-51

Cockcroft VG, Ross GJB, Peddemors VM (1991) Distribution and status of bottlenose dolphin Tursiops truncatus on the south coast of Natal, South Africa. S Afr J Mar Sci 11: 203-209

Cockcroft VG, Ross GJB, Peddemors VM, Borchers DL (1992) Estimates of abundance and undercounting of bottlenose dolphins off northern Natal, South Africa. S Afr J Wildl Res 22:102-109

Committee on Taxonomy (2012) List of marine mammal species and subspecies. Society for Marine Mammalogy, Hartford, ME. Available at www.marinemammalscience. org (accessed 7 August 2012)

COSEWIC (Committee on the Status of Endangered Wildlife in Canada) (2010) COSEWIC assessment and status report on the northern fur seal Callorhinus ursinus in Canada. COSEWIC, Ottawa

> Crespo EA, Pedraza SN, Grandi MF, Dans SL, Garaffo GV (2010) Abundance and distribution of endangered franciscana dolphins in Argentine waters and conservation implication. Mar Mamm Sci 26:17-35

D'Agrosa C, Vidal O, Graham WC (1995) Mortality of the vaquita (Phocoena sinus) in gillnet fisheries during 1993-94. Rep Int Whal Comm Spec Issue 16:283-291

D'Agrosa C, Lennert-Cody CE, Vidal O (2000) Vaquita bycatch in Mexico's artisanal gillnet fisheries: driving a small population to extinction. Conserv Biol 14: 1110-1119

> Dawson SM, Slooten E (1993) Conservation of Hector's dolphins: the case and process which led to establishment of the Banks Peninsula Marine Mammal Sanctuary. Aquat Conserv 3:207-221

Dawson SM, Slooten E (2005) Management of gillnet bycatch of cetaceans in New Zealand. J Cetacean Res Manag 7:59-64

Dayaratne P, Joseph L (1993) A study on dolphin catches in Sri Lanka. BOBP/REP/56, Bay of Bengal Programme, Madras

Desportes G, Bjørge A, Rosing-Asvid A, Waring GT (eds) (2010) Harbour seals in the North Atlantic and the Baltic. Scientific Publication 8, North Atlantic Marine Mammal Commission, Tromsø

DFO (Department of Fisheries and Oceans) (2011) Updated status of northwest Atlantic harp seals (Pagophilus groenlandicus). DFO, Canadian Science Advisory Secretariat, Science Advisory Report 2011/050, Ottawa

Dmitrieva L, Kondakov A, Oleynikov E, Kydyrmanov A and others (2011) By-catch in illegal fisheries is a major source of mortality for Caspian seals. In: Proc 19th Bienn Conf Biol Mar Mammals. Society for Marine Mammalogy, Tampa, FL, p 80 (abstract) 
Dodman T, Diop NMD, Khady S (eds) (2008) Conservation strategy for the West African manatee. United Nations Environment Programme, Nairobi, and Wetlands International Africa, Dakar

Dolar MLL, Leatherwood SJ, Wood CJ, Alava MNR, Hill CL, Aragones LV (1994) Directed fisheries for cetaceans in the Philippines. Rep Int Whal Comm 44:439-449

Dungan SZ, Riehl KN, Wee A, Wang JY (2011) A review of the impacts of anthropogenic activities on the critically endangered eastern Taiwan Strait Indo-Pacific humpback dolphins (Sousa chinensis). J Mar Anim Ecol 4:3-9

Engelhaupt D, Hoelzel AR, Nicholson C, Frantzis A and others (2009) Female philopatry in coastal basins and male dispersion across the North Atlantic in a highly mobile marine species, the sperm whale (Physeter macrocephalus). Mol Ecol 18:4193-4205

FAO (Food and Agriculture Organization) (2012) The state of world fisheries and aquaculture 2012. UN FAO, Rome

Félix F, Samaniego J (1994) Incidental catches of small cetaceans in the artisanal fisheries of Ecuador. Rep Int Whal Comm Spec Issue 15:475-480

Félix F, Samaniego J, Haase B (2007) Interacciones de cetáceos con la pesquería artesanal pelágica en Ecuador. In: Félix F (ed) Memorias del taller de trabajo sobre el impacto de las actividades antropogénicas en mamíferos marinos en el Pacífico Sudeste, Bogotá, Colombia. CPPS/ PNUMA, Guayaquil, p 50-54

Félix F, Muñoz M, Falconí J, Botero N, Haase B (2011) Entanglement of humpback whales in artisanal fishing gear in Ecuador. J Cetacean Res Manag 3(Spec Issue): 285-290

Fernández-Contreras M, Cardona L, Lockyer C, Aguilar A (2010) Incidental by-catch of short beaked common dolphins (Delphinus delphis) in the pair trawler fishery off north-western Spain. ICES J Mar Sci 67:1732-1738

Flórez-González L, Capella J (2010) Interacción pesqueríacetáceos: captura incidental en el Pacífico sur de Colombia. In: Félix F (ed) Esfuerzos para mitigar el impacto de actividades pesqueras en cetáceos en los países del Pacífico Sudeste. Comisión Permanente del Pacífico Sur, Guayaquil, p 11-17

Forney KA, Benson SR, Cameron GA (2001) Central California gillnet effort and bycatch of sensitive species, 1990-98. In: Melvin EF, Parrish JK (eds) Proc Symp Seabird Bycatch: trends, roadblocks, and solutions. AK-SG-01-01, University Alaska Sea Grant, Fairbanks, AK, p 141-160

Fowler CW (1987) Marine debris and northern fur seals: a case study. Mar Pollut Bull 18:326-335

Frantzis A (1998) Does acoustic testing strand whales? Nature 392:29

Goldsworthy S, Gales N (2008) Neophoca cinerea. In: IUCN 2011. IUCN Red List of Threatened Species, Version 2011.2 Available at www.iucnredlist.org (accessed 25 April 2012)

Goldsworthy SD, Page B (2007) A risk-assessment approach to evaluating the significance of seal bycatch in two Australian fisheries. Biol Conserv 139:269-285

Goldsworthy SD, Hamer D, Page B (2007) Assessment of the implications of interactions between furseals and sea lions and the southern rock lobster and gillnet sector of the southern and eastern scale fish and shark fishery (SESSF) in south Australia. Final Report to the Fisheries Research and Development Corporation FRDC Project No. 2005/077, South Australian Research and Development Institute, Urrbrae, SA
Goodall RNP, Schiavini ACM, Fermini C (1994) Net fisheries and net mortality of small cetaceans off Tierra del Fuego, Argentina. Rep Int Whal Comm Spec Issue 15: 295-304

Gormley AM, Slooten E, Dawson SM, Barker RJ, Rayment W, du Fresne S, Bräger S (2012) First evidence that marine protected areas can work for marine mammals. J Appl Ecol 49:474-480

Güçlüsoy H (2008) Interaction between monk seals, Monachus monachus (Hermann, 1779), and artisanal fisheries in the Foça Pilot Monk Seal Conservation Area, Turkey. Zool Middle East 43:13-20

Hall MA (1996) On by-catches. Rev Fish Biol Fish 6:319-352

Hamer DJ, Ward TM, Shaughnessy PD, Clark SR (2011) Assessing the effectiveness of the Great Australian Bight Marine Park in protecting the endangered Australian sea lion Neophoca cinerea from bycatch mortality in shark gillnets. Endang Species Res 14:203-216

Hamer DJ, Goldsworthy SD, Costa DP, Fowler SL, Page B, Sumner MD (2013) The endangered Australian sea lion extensively overlaps with and regularly becomes bycatch in demersal shark gill-nets in South Australia. Biol Conserv 157:386-400

Harkonen T, Harding KC, Wilson S, Baimukanov M, Dmitrieva L, Svensson CJ, Goodman SJ (2012) Collapse of a marine mammal species driven by human impacts. PLoS ONE 7:e43130

Heyning JE, Lewis TD (1990) Entanglements of baleen whales in fishing gear off southern California. Rep Int Whal Comm 40:427-431

> Hofman RJ (1995) The changing focus of marine mammal conservation. Trends Ecol Evol 10:462-465

Ilangakoon AD, Ratnasooriya WD, Miththapala S (2000) Species diversity, seasonal variation and capture method of small cetaceans on the west coast of Sri Lanka. Vidyodaya J Sci 9:37-51

Iñíguez MA, Hevia M, Gasparow C, Tomsin AL, Secchi ER (2003) Preliminary estimate of incidental mortality of Commerson's dolphins (Cephalorhynchus commersonii) in an artisanal fishery in La Angelina Beach and Ria Gallegos, Santa Cruz, Argentina. Lat Am J Aquat Mamm 2: 87-89

International Whaling Commission (1994) Report of the workshop on mortality of cetaceans in passive fishing nets and traps. Rep Int Whal Commn 15(Spec Issue):6-71

International Whaling Commission (2000) Report of the subcommittee on small cetaceans. J Cetacean Res Manag (Suppl 2):235-263

Isaac NJB, Turvey ST, Collen B, Waterman C, Baillie JEM (2007) Mammals on the EDGE: conservation priorities based on threat and phylogeny. PLoS ONE 2:e296

Jaaman SA, Lah-Anyi YU, Pierce GJ (2009) The magnitude and sustainability of marine mammal by-catch in fisheries in East Malaysia. J Mar Biol Assoc UK 89:907-920

Jaramillo-Legorreta A, Rojas-Bracho L, Brownell RL Jr, Read AJ, Reeves RR, Ralls K, Taylor BL (2007) Saving the vaquita: immediate action, not more data. Conserv Biol 21:1653-1655

Jefferson TA (ed) (2004) Special issue: biology and conservation of humpback dolphins (Sousa spp.). Aquat Mamm 30:1-206

Johnson A, Salvador G, Kenney J, Robbins J, Kraus S, Landry S, Clapham P (2005) Fishing gear involved in entanglements of right and humpback whales. Mar Mamm Sci 21:635-645 
Karamanlidis AA, Androukaki E, Adamantopoulou S, Chatzispyrou A and others (2008) Assessing accidental entanglement as a threat to the Mediterranean monk seal Monachus monachus. Endang Species Res 5: 205-213

Kemper CM, Pemberton D, Cawthorn M, Heinrich S and others (2003) Aquaculture and marine mammals: coexistence or conflict? In: Gales N, Hindell M, Kirkwood R (eds) Marine mammals: fisheries, tourism, and management issues. CSIRO, Collingwood, p 208-225

Knowlton AR, Kraus SD (2001) Mortality and serious injury of northern right whales (Eubalaena glacialis) in the western North Atlantic Ocean. J Cetacean Res Manag 2(Spec Issue):193-208

Knowlton A, Cooper L, Hamilton P, Marx M, Pettis H, Kraus S (2008) Analysis of scarring on North Atlantic right whales (Eubalaena glacialis). Monitoring rates of entanglement interaction 1980-2004. Contract EA133F-03-SE0323, final report, National Marine Fisheries Service, Silver Spring, MD

Knowlton AR, Hamilton PK, Marx MK, Pettis HM, Kraus SD (2012) Monitoring North Atlantic right whale Eubalaena glacialis entanglement rates: a $30 \mathrm{yr}$ retrospective. Mar Ecol Prog Ser 466:295-302

Kovacs KM, Aguilar A, Aurioles D, Burkanov V and others (2012) Global threats to pinnipeds. Mar Mamm Sci 28: 414-436

Laist DW (1996) Marine debris entanglement and ghost fishing: a cryptic and significant type of bycatch? In: Solving bycatch: considerations for today and tomorrow. Proceedings of the solving bycatch workshop. Alaska Sea Grant College Prog Rep No. 96-03, University of Alaska Fairbanks, p 33-39

Laist DW (1997) Impacts of marine debris: entanglement of marine life in marine debris including a comprehensive list of species with entanglement and ingestion records In: Coe IM, Rogers DB (eds) Marine debris: sources, impacts, and solutions. Springer, New York, NY, p 99-140

Laist DW, Coe JM, O'Hara KJ (1999) Marine debris pollution. In: Twiss JR Jr, Reeves RR (eds) Conservation and management of marine mammals. Smithsonian Institution, Washington, DC, p 342-366

Larntz K, Garrott R (1993) Analysis of 1991 bycatch of selected mammal species in the North Pacific neon squid driftnet fishery. Final contract report prepared for the National Marine Fisheries Service

Leatherwood S (1994) Re-estimation of incidental cetacean catches in Sri Lanka. Rep Int Whal Comm Spec Issue 15: 64-65

Leatherwood S, Reeves RR (1989) Marine mammal conservation and research in Sri Lanka 1985-1986. Marine Mammal Technical Report 1, United Nations Environment Program, Nairobi

Lesage V, Keays J, Turgeon S, Hurtubise S (2006) Bycatch of harbour porpoise (Phocoena phocoena) in the gillnet fisheries of the estuary and Gulf of St. Lawrence, 2000-02. J Cetacean Res Manag 8:67-78

MacMillan DC, Han J (2011) Cetacean by-catch in the Korean peninsula-by chance or by design? Hum Ecol 39:757-768

Mangel JC, Alfaro-Shigueto J, Van Waerebeek K, Cáceres C, Bearhop S, Witt MJ, Godley BJ (2010) Small cetacean captures in Peruvian artisanal fisheries: high despite protective legislation. Biol Conserv 143:136-143
Marsh H., Penrose H, Eros C, Hugues J (2002) Dugong status reports and action plans for countries and territories. UNEP/DEWA/RS.02-1, Early Warning and Assessment Report Series, United Nations Environment Programme, Nairobi

May-Collado LJ, Agnarsson I (2011) Phylogenetic analysis of conservation priorities for aquatic mammals and their terrestrial relatives, with a comparison of methods. PLoS One 6:e22562

> Meÿer MA, Best PB, Anderson-Reade MD, Cliff G, Dudley SFJ, Kirkman SP (2011) Trends and interventions in large whale entanglement along the South African coast. Afr J Mar Sci 33:429-439

Minton G, Collins T, Findlay K, Ersts P, Rosenbaum $H$, Berggren P, Baldwin R (2011) Seasonal distribution, abundance, habitat use and population identity of humpback whales in Oman. J Cetacean Res Manag 3(Spec Issue):185-198

Mitchell E (ed) (1975) Review of biology and fisheries for smaller cetaceans. J Fish Res Board Can 32:887-983

Moore JE, Cox TM, Lewison RL, Read AJ and others (2010) An interview-based approach to assess marine mammal and sea turtle captures in artisanal fisheries. Biol Conserv 143:795-805

MRAG (2012) A review of bycatch in the Indian Ocean gillnet tuna fleet focussing on India and Sri Lanka. ISSF Tech Rep 2012-05, International Seafood Sustainability Foundation, Washington, DC. http://iss-foundation.org/ wp-content/uploads/downloads/2012/05/ISSF-2012-05IO-Gillnet-Bycatch.pdf

NAMMCO (North Atlantic Marine Mammal Commission) (2000) Report of the NAMMCO Scientific Committee working group on the population status of beluga and narwhal in the North Atlantic. North Atlantic Marine Mammal Commission Annual Report 1999:153-188

NAMMCO (North Atlantic Marine Mammal Commission) (2007) NAMMCO scientific committee working group on harbour seals. In: Annual Report 2006, Vol II. NAMMCO Scientific Committee, National Progress Report. North Atlantic Marine Mammal Commission, Tromsø, p 358-408

Northridge SP (1996) A review of marine mammal bycatch observer schemes with recommendations for best practice. Report 219, Joint Nature Conservation Committee, Aberdeen

Northridge SP, Hofman RJ (1999) Marine mammal interactions with fisheries. In: Twiss JR Jr, Reeves RR (eds) Conservation and management of marine mammals. Smithsonian Institution Press, Washington, DC, p 99-199

Notarbartolo di Sciara G, Frantzis A, Bearzi G, Reeves RR (2006) Sperm whale (Physeter macrocephalus) Mediterranean subpopulation. In: Reeves RR, Notarbartolo di Sciara G (eds) The status and distribution of cetaceans in the Black Sea and Mediterranean Sea. IUCN Centre for Mediterranean Cooperation, Malaga, p 48-56

Ólafsdóttir D (2009) Report on monitoring of marine mammal bycatch in Icelandic fisheries, statistics for 2009 and review of previous information. Document NAMMCO 6/SC17/16, North Atlantic Marine Mammal Commission, Tromsø

Orphanides CD, Palka DL (2013) US Northwestern Atlantic gillnet harbor porpoise take reduction plan: trends in bycatch, compliance, and enforcement. Endang Species Res (in press) 
Ott PH, Secchi ER, Moreno IB, Danilewicz D and others (2002) Report of the working group on fishery interactions. Latin Am J Aquat Mamm 1:55-64

Page B, McKenzie J, McIntosh R, Baylis A and others (2004) Entanglement of Australian sea lions and New Zealand fur seals in lost fishing gear and other marine debris before and after government and industry attempts to reduce the problem. Mar Pollut Bull 49:33-42

Peddemors VM (1998) Delphinids of southern Africa: a review of their distribution, status and life history. J Cetacean Res Manag 1:157-165

Peddemors VM, Cockcroft VG, Best PB (1997) Exploitation of small cetaceans off South Africa: 1978-1996. Unpublished report. Document SC/49/SM34. Available from Secretariat, International Whaling Commission, Cambridge

Perrin WF, Donovan GP, Barlow J (eds) (1994) Gillnets and cetaceans: incorporating the proceedings of the symposium and workshop on the mortality of cetaceans in passive fishing nets and traps. Rep Int Whal Comm Spec Issue 15:1-629

Perrin WF, Reeves RR, Dolar MLL, Jefferson TA, Marsh H, Wang JY, Estacion J (eds) (2005) Report of the second workshop on the biology and conservation of small cetaceans and dugongs of South-East Asia. Convention on Migratory Species Tech Ser Publication 9, UNEP/CMS Secretariat, Bonn, p 1-161

Pinedo MC, Polachek T (1999) Trends in franciscana (Pontoporia blainvillei) stranding rates in Rio Grande do Sul, southern Brazil (1979-1998). J Cetacean Res Manag 1: 179-189

Pyenson ND (2009) Requiem for Lipotes: an evolutionary perspective on marine mammal extinction. Mar Mamm Sci 25:714-724

Raum-Suryan KL, Jemison LA, Pitcher KW (2009) Entanglement of Steller sea lions (Eumetopias jubatus) in marine debris: identifying causes and finding solutions. Mar Pollut Bull 58:1487-1495

Razafindrakoto Y, Andrianarivelo N, Rosenbaum HC (2004) Sightings, catches, and other records of Indo-Pacific humpback dolphins in the coastal waters of Madagascar. Aquat Mamm 30:103-110

Read AJ (2005) Bycatch and depredation In: Reynolds III JE, Perrin WF, Reeves RR, Montgomery S, Ragen TJ (eds) Marine mammal research: conservation beyond crisis. Johns Hopkins University Press, Baltimore, MD, p 5-17

Read AJ (2008) The looming crisis: interactions between marine mammals and fisheries. J Mammal 89:541-548

Read AJ (2013) Development of conservation strategies used to mitigate the by-catch of harbour porpoises in the Gulf of Maine. Endang Species Res (in press)

$>$ Read AJ, Van Waerebeek K, Reyes JC, McKinnon JS, Lehman LC (1988) The exploitation of small cetaceans in coastal Peru. Biol Conserv 46:53-70

> Read AJ, Drinker P, Northridge S (2006) Bycatch of marine mammals in U.S. and global fisheries. Conserv Biol 20: 163-169

Reeves R, Wang JY (2011) Neophocaena phocaenoides. In: IUCN 2012. IUCN Red List of Threatened Species, Version 2012.1. IUCN, Gland. Available at www.iucnred list.org (accessed 14 October 2012)

Reeves RR, Tuboku-Metzger D, Kapindi RA (1988) Distribution and exploitation of manatees in Sierra Leone. Oryx 22:75-84
Reeves RR, Leatherwood S, Jefferson TA, Curry BE, Henningsen T (1996) Amazonian manatees, Trichechus inunguis, in Peru: distribution, exploitation, and conservation status. Interciencia 21:246-254

Reeves RR, Wang JY, Leatherwood S (1997) The finless porpoise, Neophocaena phocaenoides (G. Cuvier, 1829): a summary of current knowledge and recommendations for conservation action. Asian Mar Biol 14:111-143

Reeves RR, Jefferson TA, Karczmarski L, Laidre K and others (2008a) Orcaella brevirostris. In: IUCN 2011. IUCN Red List of Threatened Species, Version 2011.2. IUCN, Gland. Available at www.iucnredlist.org (accessed 7 April 2012)

Reeves RR, Jefferson TA, Karczmarski L, Laidre K and others (2008b) Orcaella heinsohni. In: IUCN 2012. IUCN Red List of Threatened Species, Version 2012.1. IUCN, Gland. Available at www.iucnredlist.org (accessed 7 August 2012)

Reeves RR, Crespo EA, Dans S, Jefferson TA and others (2008c) Cephalorhynchus eutropia. In: IUCN 2011. IUCN Red List of Threatened Species, Version 2011.2. IUCN, Gland. Available at www.iucnredlist.org (accessed 8 April 2012)

Reeves RR, Crespo EA, Dans S, Jefferson TA and others (2008d) Cephalorhynchus commersonii. In: IUCN 2011. IUCN Red List of Threatened Species, Version 2011.2. IUCN, Gland. Available at www.iucnredlist.org (accessed 8 April 2012)

Reilly SB, Bannister JL, Best PB, Brown M and others (2008) Balaenoptera acutorostrata. In: IUCN 2012. IUCN Red List of Threatened Species, Version 2012.1. Available at www.iucnredlist.org (accessed 14 July 2012)

Reyes JC, Oporto JA (1994) Gillnet fisheries and cetaceans in the southeast Pacific. Rep Int Whal Comm Spec Issue 15:467-474

Robards MD, Reeves RR (2011) The global extent and character of marine mammal consumption by humans, 1970-2009. Biol Conserv 144:2770-2786

Robbins J (2009) Scar-based inference into Gulf of Maine humpback whale entanglement: 2003-2006. Order Number EA 133F04SE0998. Final report to Northeast Fisheries Science Center, National Marine Fisheries Service, Woods Hole, MA

Robertson BC, Chilvers BL (2011) The population decline of the New Zealand sea lion Phocarctos hookeri: a review of possible causes. Mammal Rev 41:253-275

Rogan E, Mackey M (2007) Megafauna bycatch in drift nets for albacore tuna (Thunnus alalunga) in the NE Atlantic. Fish Res 86:6-14

> Rojas-Bracho L, Reeves RR, Jaramillo-Legorreta A (2006) Conservation of the vaquita Phocoena sinus. Mammal Rev 36:179-216

Ross GJB, Cockcroft VG, Melton DA, Butterworth DS (1989) Population estimates for bottlenose dolphins Tursiops truncatus in Natal and Transkei waters. S Afr J Mar Sci 8: 119-129

Scheidat M, Gilles A, Kock KH, Siebert U (2008) Harbour porpoise Phocoena phocoena abundance in the southwestern Baltic Sea. Endang Species Res 5:215-223

Secchi ER, Zerbini AN, Bassoi M, Dalla Rosa L, Möller LM, Rocha-Campos C (1997) Mortality of franciscanas, Pontoporia blainvillei, in coastal gillnets in southern Brazil: 1994-1995. Rep Int Whal Comm 47:653-658

Secchi ER, Ott PH, Crespo EA, Kinas PG, Pedraza SN, Bordino P (2001) A first estimate of franciscana (Pontoporia 
blainvillei) abundance off southern Brazil. J Cetacean Res Manag 3:95-100

Silvani L, Gazo M, Aguilar A (1999) Spanish driftnet fishing and incidental catches in the western Mediterranean. Biol Conserv 90:79-85

Simmonds MP, Lopez-Jurado LF (1991) Whales and military sonar. Nature 351:448

Sipilä T (2003) Conservation biology of Saimaa ringed seal (Phoca hispida saimensis) with reference to other European seal populations. PhD thesis, University of Helsinki, Helsinki

Sipilä T, Hyvärinen H (1998) Status and biology of Saimaa (Phoca hispida saimensis) and Ladoga (Phoca hispida ladogensis) ringed seals. In: Heide-Jørgensen MP, Lydersen C (eds) Ringed seals in the North Atlantic. North Atlantic Marine Mammal Commission Scientific Publications Vol 1, North Atlantic Marine Mammal Commission, Tromsø, p 83-99

Skóra KE, Kuklik I (2003) Bycatch as a potential threat to harbour porpoises (Phocoena phocoena) in Polish Baltic waters. Scientific publications, Vol 5, North Atlantic Marine Mammal Commission, Tromsø, p 303-315

Slooten E (2007) Conservation management in the face of uncertainty: effectiveness of four options for managing Hector's dolphin bycatch. Endang Species Res 3:169-179

Slooten E, Dawson SM (2010) Assessing the effectiveness of conservation management decisions: likely effects of new protection measures for Hector's dolphin. Aquat Conserv 20:334-347

Smith BD, Braulik GT (2008) Platanista gangetica. In: IUCN 2011. IUCN Red List of Threatened Species, Version 2011.2. Available at www.iucnredlist.org (accessed 7 April 2012)

Song KJ, Kim ZG, Zhang CI, Kim YH (2010) Fishing gears involved in entanglements of minke whales (Balaenoptera acutorostrata) in the East Sea of Korea. Mar Mamm Sci 26:282-295

Stenson GB (2003) Harbour porpoise (Phocoena phocoena) in the North Atlantic: abundance, removals, and sustainability of removals. In: Haug T, Desportes G, Víkingsson GA, Witting L (eds) Harbour porpoises in the North Atlantic. Scientific publications, Vol 5, North Atlantic Marine Mammal Commission, Tromsø, p 271-302

Taylor BL (2005) Identifying units to conserve. In: Reynolds III JE, Perrin WF, Reeves RR, Montgomery S, Ragen TJ (eds) Marine mammal research: conservation beyond crisis. Johns Hopkins University Press, Baltimore, MD, p 149-162

Taylor BL, Martinez M, Gerrodette T, Barlow J, Hrovat YN (2007) Lessons from monitoring trends in abundance of marine mammals. Mar Mamm Sci 23:157-175

Tonay AM, Öztürk AA (2012) Historical records of cetacean fishery in the Turkish seas. J Black Sea/Mediterr Environ 18:388-399

Tregenza NJC, Berrow SD, Hammond PS, Leaper R (1997) Common dolphin, Delphinus delphis L., by-catch in bot-

Editorial responsibility: William Perrin,

La Jolla, California, USA tom set gillnets in the Celtic Sea. Rep Int Whal Comm 47: 835-839

Tudela S, Kai Kai A, Maynou F, El Andalossi M, Guglielmi P (2005) Driftnet fishing and biodiversity conservation: the case study of the large-scale Moroccan driftnet fleet operating in the Alboran Sea (SW Mediterranean). Biol Conserv 121:65-78

Turvey ST, Pitman RL, Taylor BL, Barlow J and others (2007) First human-caused extinction of a cetacean species? Biol Lett 3:537-540

Tyack PL, Zimmer WM, Moretti D, Southall BL and others (2011) Beaked whales respond to simulated and actual navy sonar. PLoS ONE 6:e17009

Van Waerebeek K, Reyes JC (1994a) Interactions between small cetaceans and Peruvian fisheries in 1988/89 and analysis of trends. Rep Int Whal Comm Spec Issue 15: 495-502

Van Waerebeek K, Reyes JC (1994b) Post-ban cetacean takes off Peru: a review. Rep Int Whal Comm Spec Issue 15:503-519

Van Waerebeek K, Van Bressem M, Felix F, Alfaro-Shigueto $\mathrm{J}$ and others (1997) Mortality of dolphins and porpoises in coastal fisheries off Peru and southern Ecuador in 1994. Biol Conserv 81:43-49

Van Waerebeek K, André M, Sequeira M, Martín V and others (1999) Spatial and temporal distribution of the minke whale, Balaenoptera acutorostrata (Lacépède, 1804), in the southern northeast Atlantic Ocean and the Mediterranean Sea, with reference to stock identity. J Cetacean Res Manag 1:223-237

Van Waerebeek K, Alfaro-Shigueto J, Montes D, Onton K, Santillan L, Van Bressem MF, Vega D (2002) Fisheries related mortality of small cetaceans in neritic waters of Peru in 1999-2001. Document SC/54/SM10. Available from Secretariat, International Whaling Commission, Cambridge

- Van Waerebeek K, Barnett L, Camara A, Cham A and others (2004) Distribution, status, and biology of the Atlantic humpback dolphin, Sousa teuszii (Kükenthal, 1892). Aquat Mamm 30:56-83

Van Waerebeek K, Ofori-Danson PK, Debrah J (2009) The cetaceans of Ghana, a validated faunal checklist. West African J Appl Ecol 15:61-90

Wang JY, Reeves R (2011) Neophocaena asiaeorientalis. In: IUCN 2012. IUCN Red List of Threatened Species, Version 2012.1. IUCN, Gland. Available at www.iucnred list.org (accessed 14 October 2012)

Waring GT, Josephson E, Maze-Foley K, Rosel PE (eds) (2011) U.S. Atlantic and Gulf of Mexico Marine Mammal Stock Assessments - 2010. NOAA Tech Memo NMFS NE 219

Woodley TH, Lavigne DM (1991) Incidental capture of pinnipeds in commercial fishing gear. Technical Report 9101, International Marine Mammal Association, Guelph

Zhu Q (2012) Gray whale bycaught in Pingtan, China. Cetoken Newsl 29 2012.2.15:1-8 (in Chinese)

Submitted: August 22, 2012; Accepted: November 21, 2012 Proofs received from author(s): February 24, 2013 